\title{
Same-sign charged Higgs boson pair production in bosonic decay channels at the HL-LHC and HE-LHC
}

\author{
Abdesslam Arhrib, ${ }^{1,2}$ Kingman Cheung, ${ }^{2,3,4}$ and Chih-Ting Lu $\odot^{5}$ \\ ${ }^{1}$ Département de Mathématique, Faculté des Sciences et Techniques, \\ Université Abdelmalek Essaadi, B. 416 Tangier, Morocco \\ ${ }^{2}$ Physics Division, National Center for Theoretical Sciences, Hsinchu, Taiwan 300 \\ ${ }^{3}$ Department of Physics, National Tsing Hua University, Hsinchu 300, Taiwan \\ ${ }^{4}$ Division of Quantum Phases and Devices, School of Physics, Konkuk University, \\ Seoul 143-701, Republic of Korea \\ ${ }^{5}$ School of Physics, KIAS, Seoul 130-722, Republic of Korea
}

(Received 27 November 2019; accepted 4 November 2020; published 25 November 2020)

\begin{abstract}
The same-sign charged Higgs pair production via vector-boson scattering is a useful probe of the mass spectrum among the heavier scalar, pseudoscalar, and charged Higgs bosons in two-Higgs-doublet models. It has been shown that the production cross section scales as the square of the mass difference $\Delta m=$ $\left(m_{H^{0}}-m_{A^{0}}\right)$ in the alignment limit $(\cos (\beta-\alpha)=0)$. We study the potential measurement of this samesign charged Higgs boson pair production at the high-luminosity LHC and the proposed $27 \mathrm{TeV} p p$ collider, with emphasis in the decay channel $H^{ \pm} H^{ \pm} \rightarrow\left(W^{ \pm} A^{0}\right)\left(W^{ \pm} A^{0}\right)$, which is in general the dominant mode when the charged Higgs mass is above the $W^{ \pm} A^{0}$ threshold. We also examine the current allowed parameter space taking into account the theoretical constraints on the model, the electroweak precision test measurements, $B$ decays, and direct searches in the $H^{ \pm} \rightarrow \tau^{ \pm} \nu_{\tau}$ and $H^{ \pm} \rightarrow W^{ \pm} A^{0} \rightarrow\left(\ell^{ \pm} \nu_{\ell}\right)(\mu \mu)$.
\end{abstract}

DOI: 10.1103/PhysRevD.102.095026

\section{INTRODUCTION}

Since the discovery of a Higgs-like particle at the CERN Large Hadron Collider (LHC) in 2012, there have been many theoretical and phenomenological studies dedicated to nonminimal Higgs sector models that can explain the observed Higgs-like particle and account for some weakness of the Standard Model (SM). One common feature of many extensions of the minimal Higgs sector is the presence of extra neutral Higgs bosons as well as singly charged Higgs bosons in the physical spectrum. Therefore, the discovery of charged Higgs bosons would be an unambiguous sign of physics beyond the SM. One of the most popular models with extended Higgs sector is the two-Higgs-doublet model (2HDM) [1,2] in which one introduces two-Higgs-doublet fields to break the $S U_{L}(2) \times$ $U_{Y}(1)$ symmetry down to the $U(1)_{\text {em }}$ symmetry. In order to avoid tree-level flavor-changing neutral current (FCNC) couplings, one can advocate a natural flavor conservation imposed by a discrete $Z_{2}$ symmetry [3]. Depending on the Higgs and fermion field transformations under the $Z_{2}$, one

Published by the American Physical Society under the terms of the Creative Commons Attribution 4.0 International license. Further distribution of this work must maintain attribution to the author(s) and the published article's title, journal citation, and DOI. Funded by SCOAP . can have a number of Yukawa textures for the fermion sector, denoted by type-I, -II, -X, and -Y 2HDMs. After electroweak symmetry breaking driven by the two Higgs fields takes place, the physical spectrum of the model consists of two $C P$-even Higgs bosons $h^{0}, H^{0}$ (one of them could be identified as the observed $125 \mathrm{GeV}$ Higgs-like particle), a $C P$-odd Higgs boson $A^{0}$ and a pair of charged Higgs bosons $H^{ \pm}$.

At hadron colliders, charged Higgs bosons can be produced in a number of channels. An important source of light charged Higgs bosons is from $t \bar{t}$ production, followed by the top decay into a charged Higgs boson and a bottom quark if kinematically allowed. Other important mechanisms for singly charged Higgs boson production are the QCD processes $g b \rightarrow t H^{-}$and $g g \rightarrow t \bar{b} H^{-}$[4]. We refer to Ref. [5] for an extensive review on charged Higgs phenomenology. Charged Higgs bosons have been searched for in the past at both LEP [6] and Tevatron [7]. An upper limit of the order of $80 \mathrm{GeV}$ has been set at LEP experiments both from fermionic and bosonic decays $H^{ \pm} \rightarrow W^{ \pm} A^{0}$ [6]. While at the Tevatron, a search for the charged Higgs from top decay had been performed in various decay channels of $H^{ \pm}$and limits on $B\left(t \rightarrow H^{+} b\right)$ have been set [7]. At the LHC, one can search for light $H^{ \pm}$from top decay and for heavy $H^{ \pm}$from $g b \rightarrow t H^{-}$and $g g \rightarrow t \bar{b} H^{-}$. Light charged Higgs boson $\left(\leq m_{t}-m_{b}\right)$ would decay dominantly into $\tau \nu_{\tau}$, $c \bar{s}$, or $c \bar{b}$ final states. In case of light pseudoscalar boson $A^{0}$, 
$H^{ \pm}$can also decay into $W^{ \pm} A^{0}$. However, a heavy $H^{+}$can also decay into $t \bar{b}, W^{+} h^{0}, W^{+} A^{0}$, or $W^{+} H^{0}$ if kinematically open. Both at the LHC Run-1 and Run-2, ATLAS and CMS had already set exclusion limits on $B\left(t \rightarrow b H^{+}\right) \times B\left(H^{+} \rightarrow\right.$ $\tau^{+} \nu_{\tau}$ ) [8-13], which can be used to set limits on $\tan \beta$ for a given charged Higgs mass less than $\leq m_{t}-m_{b}$. Moreover, from $t \rightarrow b H^{+}$, there has been also a search for $\mathrm{H}^{+} \rightarrow c \bar{s}$ channel both by ATLAS and CMS [14] at 7 and $8 \mathrm{TeV}$. The limit obtained on $B\left(t \rightarrow b H^{+}\right)$is rather weak compared to the $\tau \nu_{\tau}$ mode. Both ATLAS and CMS also searched for $H^{ \pm} \rightarrow t b$ decay, in which no $H^{ \pm}$signal was observed and upper limits on the $\sigma\left(p p \rightarrow t b H^{ \pm}\right) \times B\left(H^{ \pm} \rightarrow t b\right)$ are set [15-18].

In the 2HDM, it has been shown [19-21] that the charged Higgs boson can decay dominantly into the bosonic final state $H^{ \pm} \rightarrow W^{ \pm} A^{0}$ when kinematically open. Other models beyond SM could also have similar features such as 2HDM with singlet scalars [22] and also the nextto-minimal supersymmetric Standard Model [23]. At LEPII [24], pair-produced charged Higgs bosons have been searched in various final states, including $\tau^{+} \nu_{\tau} \tau^{-} \bar{\nu}_{\tau}$, $c \bar{s} \bar{c} s, c \bar{s} \tau^{-} \bar{\nu}_{\tau}, W^{*} A W^{*} A$, and $W^{*} A \tau^{-} \bar{\nu}_{\tau}$, and an upper limit of the order $80 \mathrm{GeV}$ was set on the charged Higgs mass. Recently, CMS also performed a search for such bosonic decays of the charged Higgs [25]. The study was only dedicated to light charged Higgs produced from top decay followed by $H^{ \pm} \rightarrow W^{ \pm} A^{0}$, where $A^{0}$ decays into a pair of muons and $W^{ \pm}$decays into a charged lepton $(e, \mu)$ and a neutrino. Assuming that $H^{ \pm}$decays $100 \%$ into $W^{ \pm} A^{0}$ and $B\left(A^{0} \rightarrow \mu \mu\right)=3 \times 10^{-4}$, CMS set a new and first limit from bosonic decay of $H^{ \pm}$on $B\left(t \rightarrow b H^{+}\right)$.

Recently, Ref. [26] proposed a new mechanism where a pair of same-sign charged Higgs bosons is produced via vector-boson fusion (VBF) at hadron colliders. Such a process can shade some light on the global symmetry of the underlying scalar potential. Assuming that the charged Higgs bosons decay into $\tau \nu_{\tau}$ or $t b$, Ref. [26] evaluated the signal and the SM backgrounds, and discussed the feasibility of the new process both for the high-luminosity LHC (HL-LHC) with $14 \mathrm{TeV}$ center of mass energy and also for the future high-energy LHC (HE-LHC) $27 \mathrm{TeV}$.

In this work, motivated by the recent CMS search for the bosonic decay $H^{ \pm} \rightarrow W^{ \pm} A^{0}$, we investigate same-sign charged Higgs production from VBF, followed by bosonic decays of the charged Higgs boson,

$$
p p \rightarrow j j W^{ \pm *} W^{ \pm *} \rightarrow j j H^{ \pm} H^{ \pm} \rightarrow j j\left(W^{ \pm} A^{0}\right)\left(W^{ \pm} A^{0}\right)
$$

in type-I and type-X 2HDMs. We calculate the signal and various SM backgrounds, and estimate the sensitivity at the HL-LHC as well as for the future hadron collider HE-LHC with $27 \mathrm{TeV}$ center of mass energy. Another important observation that motivates our work is because the fermionic production modes for these new scalars are highly suppressed by large $\tan \beta$ in both type-I and type-X $2 \mathrm{HDMs}$, the discovery of these new scalars via fermionic modes is indeed challenging at the LHC. Therefore, we are exploring the bosonic decay mode of the charged scalar $H^{ \pm} \rightarrow W^{ \pm} A^{0}$, which dominates for $\tan \beta>5$.

We should emphasize, instead of studying each new scalar (or two of them) in different processes separately, the novel process we consider here involves the effects of all new scalar masses. It means that we have the chance to simultaneously test the whole mass spectrum in the 2HDM for some specific mass relations via a single process. Finally, we show that the mass spectrum of $m_{A^{0}}=63-100 \mathrm{GeV}$ and $\Delta m \equiv$ $m_{H^{0}}-m_{A^{0}}=200-250 \mathrm{GeV}$ in type-I and type-X 2HDMs can be explored at the HE-LHC in the future after the accelerator and detector are further upgraded.

The strategy in this work is twofold. If any new scalar has been discovered in the future, the signal process considered here serves to confirm or rule out some specific mass spectra in the 2HDMs. On the other hand, if we do not find any positive evidence of new scalars in the future, the signal process in this study can also help to clarify which kind of mass spectra in 2HDMs is not preferred.

The organization is as follows. In the next section, we describe briefly the 2HDMs and relevant interactions. In Sec. III, we discuss the constraints on the model from theoretical requirements, electroweak precision test measurements, $B$ decays, and direct searches. In Sec. IV, we calculate the same-sign charged Higgs boson production cross sections and perform the signal-background analysis. We conclude in Sec. V.

\section{BRIEF REVIEW OF TWO-HIGGS-DOUBLET MODELS}

Many beyond Standard Model process an extended Higgs sector with more Higgs doublets, Higgs singlets, Higgs triplets, or a mixture of all. One of the simplest, popular, and well-motivated extensions of the SM is the two-Higgs-doublet model. A variety of which can be used in the minimal supersymmetric Standard Model (MSSM). In 2HDM, two-Higgs-doublet fields $\Phi_{1,2}$ with hypercharge $Y_{\Phi_{1,2}}=1 / 2$ are introduced. The most general renormalizable scalar potential, which respects the $S U_{L}(2) \otimes U_{Y}(1)$ gauge symmetry, has the following form:

$$
\begin{aligned}
\mathrm{V}\left(\Phi_{1}, \Phi_{2}\right)= & m_{11}^{2} \Phi_{1}^{\dagger} \Phi_{1}+m_{22}^{2} \Phi_{2}^{\dagger} \Phi_{2}+\left(m_{12}^{2} \Phi_{1}^{\dagger} \Phi_{2}+\text { H.c. }\right) \\
& +\frac{\lambda_{1}}{2}\left(\Phi_{1}^{\dagger} \Phi_{1}\right)^{2}+\frac{\lambda_{2}}{2}\left(\Phi_{2}^{\dagger} \Phi_{2}\right)^{2}+\lambda_{3} \Phi_{1}^{\dagger} \Phi_{1} \Phi_{2}^{\dagger} \Phi_{2} \\
& +\lambda_{4} \Phi_{1}^{\dagger} \Phi_{2} \Phi_{2}^{\dagger} \Phi_{1}+\left[\frac{\lambda_{5}}{2}\left(\Phi_{1}^{\dagger} \Phi_{2}\right)^{2}+\text { H.c. }\right] \\
& +\left[\left(\lambda_{6}\left(\Phi_{1}^{\dagger} \Phi_{1}\right)+\lambda_{7}\left(\Phi_{2}^{\dagger} \Phi_{2}\right)\right)\left(\Phi_{1}^{\dagger} \Phi_{2}\right)+\text { H.c. }\right]
\end{aligned}
$$

where $m_{11}^{2}, m_{22}^{2}$, and $\lambda_{1,2,3,4}$ are real, while $m_{12}^{2}$ and $\lambda_{5,6,7}$ could be complex for $C P$ violation purpose. If we require that the potential to be invariant under a discrete $Z_{2}$ 
symmetry $\Phi_{1} \rightarrow \Phi_{1}, \Phi_{2} \rightarrow-\Phi_{2}$ which is needed for natural flavor conservation in the Yukawa sector (see discussion below), this would lead to $\lambda_{6,7}=0$. One can still allow a soft violation of the discrete symmetry by a dimension-two terms $m_{12}^{2}$. In what follows, we assume that $\lambda_{6,7}=0$ and $m_{12}^{2} \neq 0$.

Assuming that both $\Phi_{1}$ and $\Phi_{2}$ acquire a vacuum expectation value (VEV) $v_{1,2}$ that can induce electroweak symmetry breaking, the two complex scalar $S U_{L}(2)$ doublets can be decomposed according to

$$
\Phi_{i}=\left(\begin{array}{c}
\phi_{i}^{+} \\
\left(v_{i}+\rho_{i}+i \eta_{i}\right) / \sqrt{2}
\end{array}\right), \quad i=1,2 .
$$

The mass eigenstates for the Higgs sector are obtained by orthogonal transformations,

$$
\begin{aligned}
\left(\begin{array}{c}
\phi_{1}^{ \pm} \\
\phi_{2}^{ \pm}
\end{array}\right) & =R_{\beta}\left(\begin{array}{c}
G^{ \pm} \\
H^{ \pm}
\end{array}\right), \quad\left(\begin{array}{c}
\rho_{1} \\
\rho_{2}
\end{array}\right)=R_{\alpha}\left(\begin{array}{c}
H^{0} \\
h^{0}
\end{array}\right), \\
\left(\begin{array}{l}
\eta_{1} \\
\eta_{2}
\end{array}\right) & =R_{\beta}\left(\begin{array}{c}
G^{0} \\
A^{0}
\end{array}\right),
\end{aligned}
$$

with the generic form $(\theta=\alpha, \beta)$,

$$
R_{\theta}=\left(\begin{array}{cc}
\cos \theta & -\sin \theta \\
\sin \theta & \cos \theta
\end{array}\right)
$$

From the 8 degrees of freedom initially present in the two scalar doublets, three of them, namely, the Goldstone bosons $G^{ \pm}$and $G^{0}$, are eaten by the longitudinal component of $W^{ \pm}$ and $Z^{0}$, respectively. The remaining 5 degrees of freedom should manifest as physical Higgs bosons: two $C P$-even $H^{0}$ and $h^{0}$, one $C P$-odd $A^{0}$, and a pair of charged scalars $H^{ \pm}$. In the $C P$ conserving case, the above potential contains ten parameters (including the VEVs of the Higgs doublets). $m_{11}^{2}$ and $m_{22}^{2}$ can be eliminated by the use of the two minimization conditions. One of the VEVs can be traded from the $W$ mass as a function of the ratio $\tan \beta \equiv v_{2} / v_{1}$. We are then left with seven independent parameters which can be taken as the four physical masses $m_{h}, m_{H}, m_{A}$, and $m_{H \pm}, C P$-even mixing angle $\alpha, \tan \beta$, and $m_{12}^{2}$.

In the Yukawa sector, it is well known that if we assume that both Higgs doublets couple to all fermions we will end up with large FCNCs mediated by the neutral Higgs scalars at tree level. In order to avoid such FCNCs, a discrete $Z_{2}$ symmetry (where $\Phi_{1} \rightarrow \Phi_{1}$ and $\Phi_{2} \rightarrow-\Phi_{2}$ ) is imposed [3]. Note that in the above potential, the $Z_{2}$ symmetry is only violated by the dimension-two term involving $m_{12}^{2}$. Depending on the $Z_{2}$ charge assignment to the lepton and quark fields $[2,27,28]$, one can have four different types of Yukawa textures. ${ }^{1}$ In the type-I model, only the second doublet $\Phi_{2}$ interacts with all the fermions like in the SM,

\footnotetext{
${ }^{1}$ Here we follow the same notation as in Ref. [27].
}

TABLE I. Yukawa coupling coefficients $\xi_{f}^{A^{0}}$ to the up-quarks, down-quarks, and the charged leptons $(f=u, d, l)$ in the four 2HDM types.

\begin{tabular}{lcrr}
\hline \hline Type & $\xi_{u}^{A^{0}}$ & \multicolumn{1}{c}{$\xi_{d}^{A^{0}}$} & \multicolumn{1}{c}{$\xi_{l}^{A^{0}}$} \\
\hline I & $\cot \beta$ & $-\cot \beta$ & $-\cot \beta$ \\
II & $\cot \beta$ & $\tan \beta$ & $\tan \beta$ \\
$\mathrm{X}$ & $\cot \beta$ & $-\cot \beta$ & $\tan \beta$ \\
$\mathrm{Y}$ & $\cot \beta$ & $\tan \beta$ & $-\cot \beta$ \\
\hline \hline
\end{tabular}

while in the type-II model $\Phi_{1}$ interacts with the charged leptons and down-type quarks and $\Phi_{2}$ interacts with uptype quarks. In the type-X (lepton-specific) model, charged leptons couple to $\Phi_{1}$ while all the quarks couple to $\Phi_{2}$. Finally, in the type-Y (flipped) model, down-type quarks acquire masses from their couplings to $\Phi_{1}$ while charged leptons and up-type quarks couple to $\Phi_{2}$. The most general Yukawa interaction can be written as follows [2]:

$-\mathcal{L}_{\text {Yukawa }}^{2 \mathrm{HDM}}=\bar{Q}_{L} Y_{u} \tilde{\Phi}_{2} u_{R}+\bar{Q}_{L} Y_{d} \Phi_{d} d_{R}+\bar{L}_{L} Y_{\ell} \Phi_{\ell} \ell_{R}+$ H.c.,

where $\Phi_{d, l}(d, l=1,2)$ represent $\Phi_{1}$ or $\Phi_{2}, Y_{f}(f=u, d$ or $\ell$ ) stand for $3 \times 3$ Yukawa matrices, and $\tilde{\Phi}_{2}=i \sigma_{2} \Phi_{2}^{\star}$.

Writing the Yukawa interactions Eq. (5) in terms of the mass eigenstates of the neutral and charged Higgs bosons yields

$$
\begin{aligned}
-\mathcal{L}_{\text {Yukawa }}^{2 \mathrm{HDM}}= & \sum_{f=u, d, \ell} \frac{m_{f}}{v}\left(\xi_{f}^{h^{0}} \bar{f} f h^{0}+\xi_{f}^{H^{0}} \bar{f} f H^{0}-i \xi_{f}^{A^{0}} \bar{f} \gamma_{5} f A^{0}\right) \\
& +\left\{\frac{\sqrt{2} V_{u d}}{v} \bar{u}\left(m_{u} \xi_{u}^{A^{0}} \mathrm{P}_{L}+m_{d} \xi_{d}^{A^{0}} \mathrm{P}_{R}\right) d H^{+}\right. \\
& \left.+\frac{\sqrt{2} m_{\ell} \xi_{\ell}^{A^{0}}}{v} \overline{\nu_{L}} \ell_{R} H^{+}+\text {H.c. }\right\}
\end{aligned}
$$

where $v^{2}=v_{1}^{2}+v_{2}^{2}=\left(2 \sqrt{2} G_{F}\right)^{-1} ; P_{R}$ and $P_{L}$ are the right- and left-handed projection operators, respectively. The coefficients for $\xi_{f}^{A^{0}}(f=u, d, l)$ in the four 2HDM types, which are relevant to this work, are given in Table I.

\section{CONSTRAINTS}

We consider both theoretical and experimental constraints on 2HDMs.

\section{A. Theoretical and electroweak precision constraints}

For theoretical constraints, we take into account all set of tree-level perturbative unitarity conditions [29-31]. We use the unitarity constraints from Ref. [31] and require that the eigenvalues of the scattering amplitudes satisfy the original Lee-Quigg-Thacker bound [29]. We also require that all $\lambda_{i}$ 's remain perturbative. Moreover, we demand that the potential remains bounded from below when the Higgs fields become large in any direction of the field space [2], which results in the following set of constraints: 

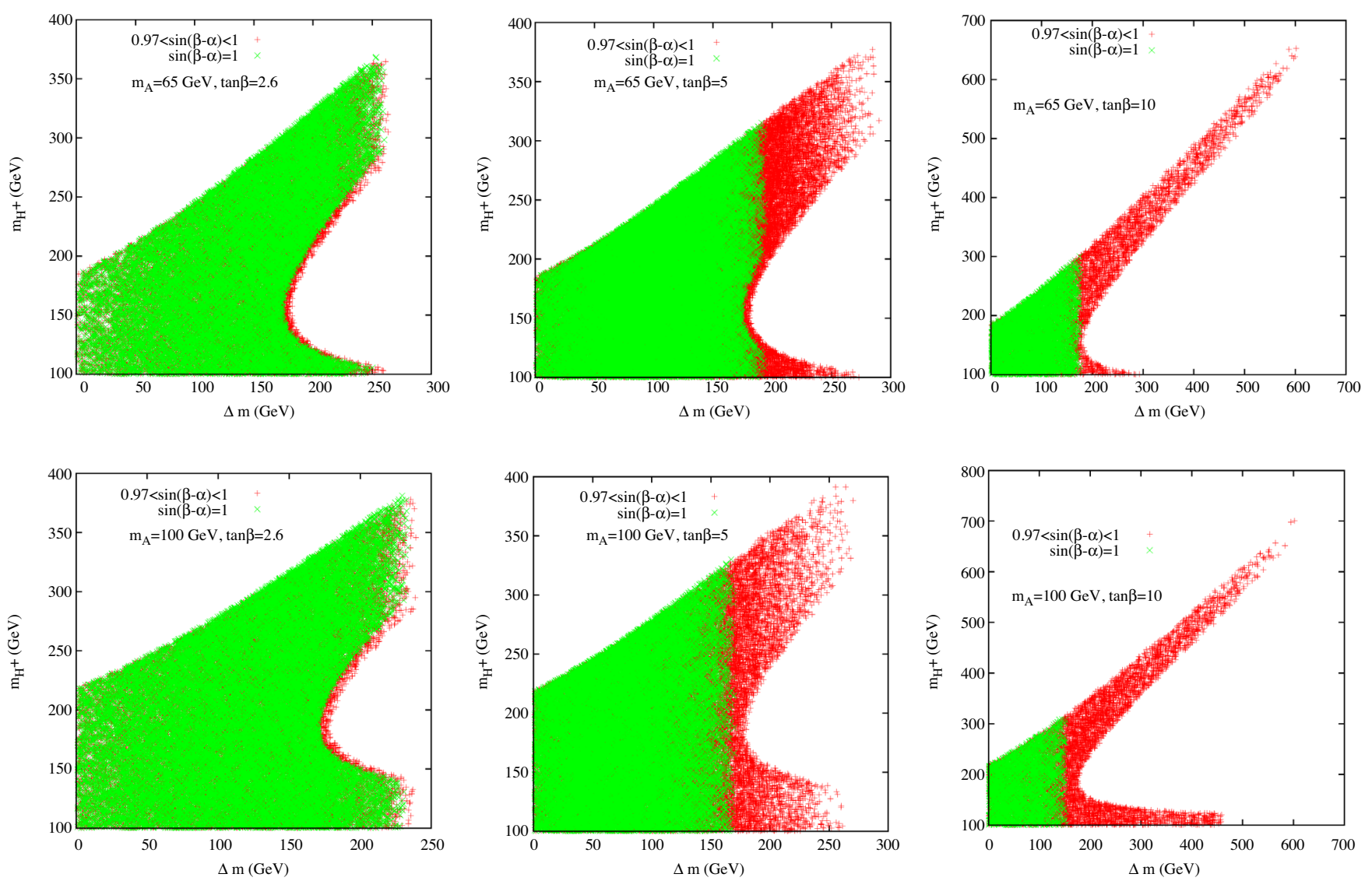

FIG. 1. The allowed parameter space in the plane of $\left(\Delta m \equiv m_{H^{0}}-m_{A^{0}}, m_{H^{ \pm}}\right)$due to the constraints from the oblique $S$ and $T$ parameters, and all theoretical constraints. The upper panels are for $m_{A^{0}}=65 \mathrm{GeV}$, while the lowers for $m_{A^{0}}=100 \mathrm{GeV}$, in which $\tan \beta=2.6$ (left), 5 (middle), and 10 (right) are shown. The green points are right at the alignment $\operatorname{limit} \sin (\beta-\alpha)=1$, while the red points satisfy $0.97<\sin (\beta-\alpha)<1$ (near-alignment limit).

$$
\begin{aligned}
& \lambda_{1}>0, \quad \lambda_{2}>0, \\
& \sqrt{\lambda_{1} \lambda_{2}}+\lambda_{3}+\min \left(0, \lambda_{4}+\lambda_{5}, \lambda_{4}-\lambda_{5}\right)>0 .
\end{aligned}
$$

For experimental constraints, we can further divide them into indirect and direct searches. The indirect searches mainly arise from electroweak precision observables (EWPOs) and flavor physics. The EWPOs can be represented by a set of oblique parameters $S, T$, and $U$. From 2018, Particles Data Group review [32] with a fixed $U=0$, the best fit of $S, T$ parameters can be represented as $S=0.02 \pm 0.07$ and $T=0.06 \pm 0.06$. We emphasize that the $T$ parameter, which is related to the amount of isospin violation, is sensitive to the mass splitting among $H^{ \pm}, H^{0}$, and $A^{0}$. It will restrict the allowed mass spectrum for the scalars in our analysis below. In order to fulfill the $T$ constraint in the 2HDM, the spectrum should be chosen close to the approximate custodial symmetry [33], which is satisfied in one of the following limits: (i) $m_{H^{ \pm}}=m_{A^{0}}$, (ii) $m_{H^{ \pm}}=m_{H^{0}}$ together with $\sin (\beta-\alpha)=1$, or (iii) $m_{H^{ \pm}}=m_{H^{0}}$ together with $\cos (\beta-\alpha)=1$.

As mentioned before, the oblique parameter $T$ is highly sensitive to the mass splitting among $H^{ \pm}, H^{0}$, and $A^{0}{ }^{2}$ In order to obtain the allowed parameter space for the mass of charged Higgs boson and the mass splitting $\Delta m=m_{H^{0}}-m_{A^{0}}$, we consider all the above theoretical constraints and $3 \sigma$ allowed regions of the $S$ and $T$ parameters in Fig. 1 for $\tan \beta=2.6,5$, and 10 with $m_{A^{0}}=65$ and $100 \mathrm{GeV}$, respectively. We also scan on $m_{12}^{2}$ in the following range $\left[0,10^{6}\right] \mathrm{GeV}^{2}$ in order to satisfy the perturbative unitarity and vacuum stability constraints for a fixed set of physical masses and mixings. We notice that, in our parameter space, the $S$ parameter is always within the best-fit range while the $T$ parameter severely constrains the splitting between $m_{A^{0}}$ and $m_{H^{ \pm}}$, and also $\Delta m$.

For $\tan \beta=2.6$, there is no significant difference in the allowed region between the alignment limit $\sin (\beta-\alpha)=1$

\footnotetext{
${ }^{2}$ Here we assume the SM-like Higgs boson is the lightest $C P$ even scalar $\left(m_{H^{0}}>m_{h^{0}}\right)$. For the reversed case $m_{H^{0}}=125 \mathrm{GeV}$ and $m_{h^{0}}<m_{H^{0}}$, with another near-alignment limit of $\cos (\beta-\alpha)$ one can also consider another process

$$
p p \rightarrow j j W^{ \pm *} W^{ \pm *} \rightarrow j j H^{ \pm} H^{ \pm} \rightarrow j j\left(W^{ \pm} h^{0}\right)\left(W^{ \pm} h^{0}\right),
$$

which is similar to the process considered in this work.
} 
and the near-alignment limit $0.97<\sin (\beta-\alpha)<1$. In the case where $\tan \beta=5$, one can see that $\Delta m$ is constrained to be less than about $200 \mathrm{GeV}$ in the exact alignment limit. This cut on $\Delta m$ is in fact due to the vacuum stability constraints in Eq. (7), where either $\lambda_{1}$ or the third constraint in Eq. (7) becomes quickly negative. While in the case near-alignment limit $0.97<\sin (\beta-\alpha)<1$, which allows the vacuum stability to be fulfilled and $\Delta m$ can reach up to $280 \mathrm{GeV}$. This correlation between vacuum stability and $\sin (\beta-\alpha) \in[0.97,1]$ is also observed in the case $\tan \beta=$ 10 and is even more pronounced where one can see that $\Delta m$ can reach up to $600 \mathrm{GeV}$. The parameter space can be divided into two parts. The first region of parameter space is for light $H^{ \pm}$. Once $m_{H^{ \pm}} \sim m_{A^{0}}$, the mass splitting $\Delta m$ can be as large as $300-450 \mathrm{GeV}$. The second region is for heavy $H^{ \pm}$. When $m_{H^{ \pm}} \sim m_{H^{0}}$, the mass splitting $\Delta m$ can be extended to about $600 \mathrm{GeV}$ for $\tan \beta=10$. While in the case $\tan \beta=5$, the maximum mass splitting $\Delta m$ is less than $200 \mathrm{GeV}$ in the alignment limit $\sin (\beta-\alpha)=1$ and could be extended to more than $250 \mathrm{GeV}$ for $0.97<\sin (\beta-\alpha)<1$. We stress that even in the case where $\Delta m$ is rather small, the $T$ parameter severely constrains the charged Higgs mass to be less than about $200 \mathrm{GeV}$ for $\tan \beta=2.6,5$, and 10 .

\section{B. B-physics constraints}

The most severe constraints in flavor physics are due to the measurements of $B\left(B \rightarrow X_{s} \gamma\right), B\left(B_{d, s} \rightarrow \mu^{+} \mu^{-}\right)$, and
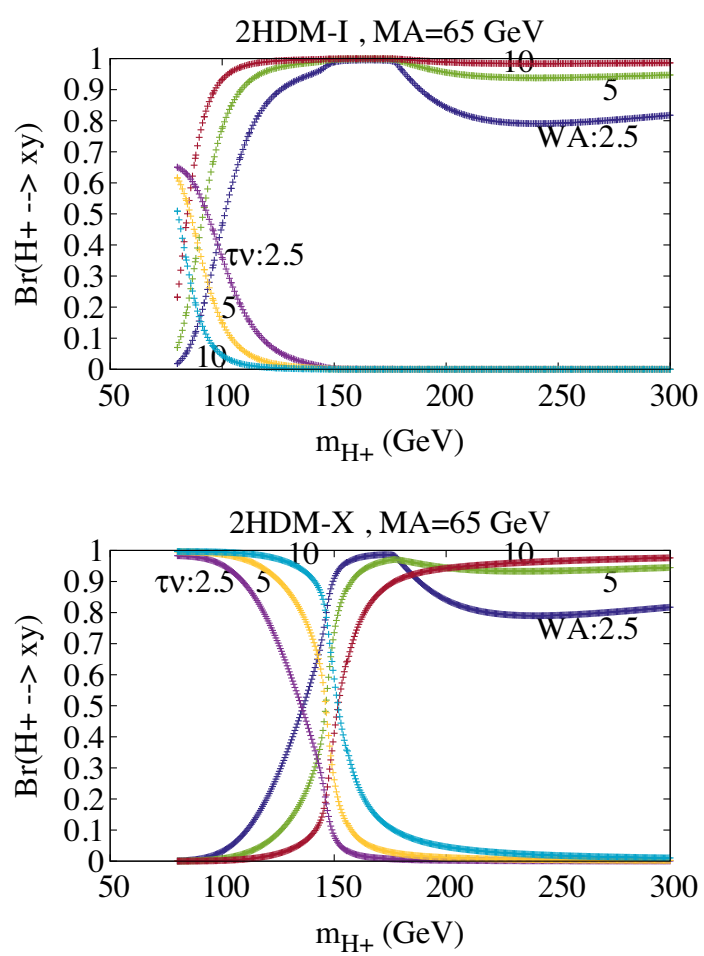

$\Delta m_{s}$ of $B$ mesons. For $B\left(B \rightarrow X_{s} \gamma\right)$, according to the latest analysis by [34], we have the following:

(i) In type-II and type-Y 2HDM, the $b \rightarrow s \gamma$ constraint forces the charged Higgs mass to be heavier than $580 \mathrm{GeV}[34,35]$ for any value of $\tan \beta \geq 1$.

(ii) In type-I and type-X $2 \mathrm{HDM}$, charged Higgs with mass as low as $\sim 100-200 \mathrm{GeV}[34,36]$ is still allowed as long as $\tan \beta \geq 2$.

For other B-physics observables, we refer to the recent analysis [37], in which they also included $\Delta m_{s}$ and $B_{d, s} \rightarrow$ $\mu^{+} \mu^{-}$. For a light charged Higgs boson, $100<m_{H^{ \pm}}<$ $200 \mathrm{GeV}$, of interest in this study, one can conclude from [37] that $\tan \beta \geq 3$ is allowed for $2 \mathrm{HDM}$ types I and $\mathrm{X}$.

\section{C. $H^{ \pm}$and $A^{0}$ branching ratios and direct searches}

Before discussing the constraints coming from direct searches, we first show the branching ratios of $H^{ \pm}$and $A^{0}$ in both 2HDM types I and $\mathrm{X}$ in the following subsection. Calculations of these branching ratios are performed using the public code 2HDMC [38].

\section{Branching ratios of $\mathrm{H}^{ \pm}$and $A^{\mathbf{0}}$}

We illustrate in Fig. 2 the branching ratios of the charged Higgs boson for both $2 \mathrm{HDM}$ types I and X. It is clear that once the bosonic decay mode $H^{ \pm} \rightarrow W^{ \pm} A^{0}$ is open, it can be the dominant decay mode and both $B\left(H^{+} \rightarrow t \bar{b}\right)$ and $B\left(H^{ \pm} \rightarrow \tau^{ \pm} \nu_{\tau}\right)$ are highly suppressed.
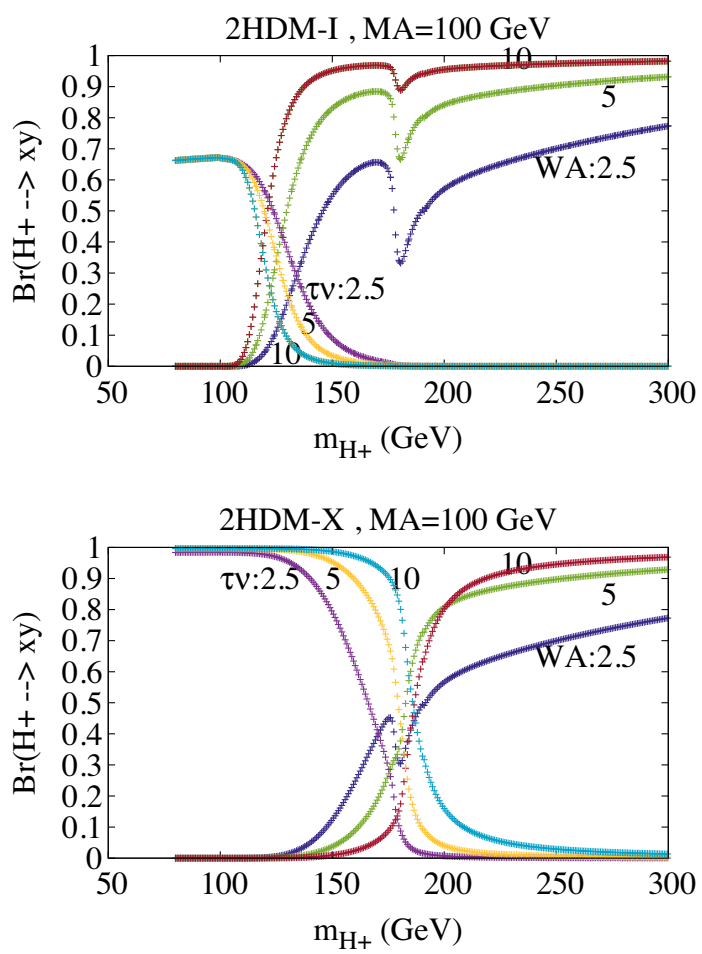

FIG. 2. Branching fractions of the charged Higgs boson versus $m_{H^{ \pm}}$for type-I 2HDM with $m_{A^{0}}=65 \mathrm{GeV}$ (upper-left panel), $m_{A^{0}}=100 \mathrm{GeV}$ (upper-right panel), and for type-X $2 \mathrm{HDM}$ with $m_{A^{0}}=65 \mathrm{GeV}$ (lower-left panel), $m_{A^{0}}=100 \mathrm{GeV}$ (lower-right panel). The alignment limit $\sin (\beta-\alpha)=1$ is assumed. 

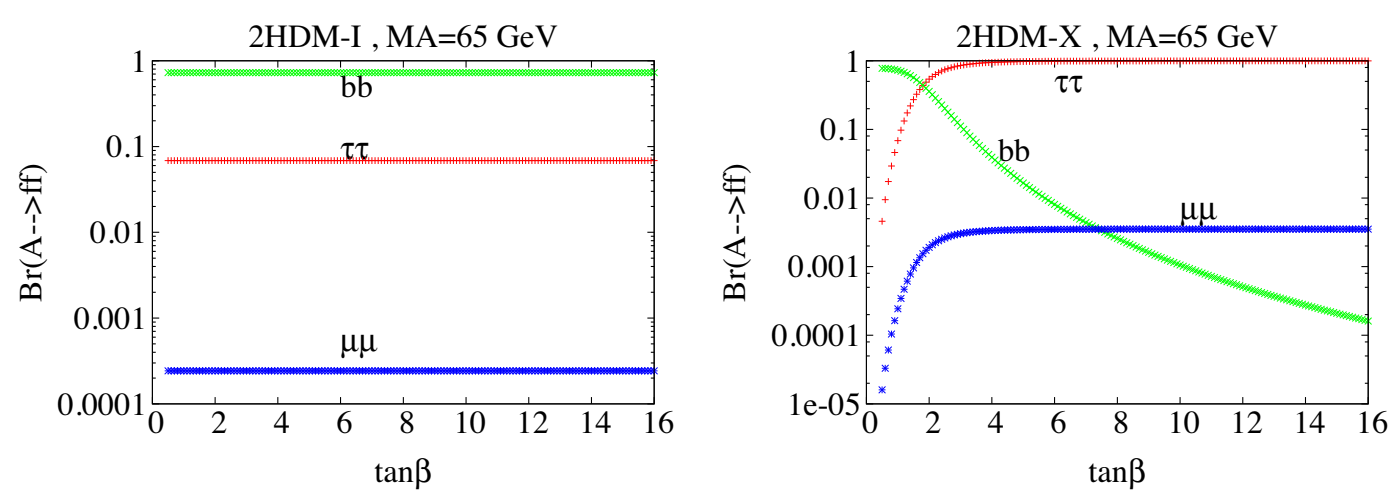

FIG. 3. Branching fractions of the $C P$-odd Higgs boson $A^{0}$ versus $\tan \beta$ for $m_{A^{0}}=65 \mathrm{GeV}$ in type-I $2 \mathrm{HDM}$ (left panel) and type-X 2HDM (right panel).

In type I, one can see that the full dominance of the bosonic decay needs $\tan \beta>5$ which reduces the $H^{ \pm} \rightarrow$ $\tau^{ \pm} \nu_{\tau}$ and $H^{ \pm} \rightarrow t b$ channels. The decay channel $H^{ \pm} \rightarrow$ $W^{ \pm} h^{0}$ is vanishing because $H^{ \pm} W^{\mp} h^{0}$ coupling is proportional to $\cos (\beta-\alpha) \approx 0$. In $2 \mathrm{HDM}$ type $\mathrm{X}$, the coupling $H^{ \pm} \tau^{\mp} \nu_{\tau}$ is proportional to $\tan \beta$ and since we assume that $\tan \beta \geq 2.5$, the $\tau \nu_{\tau}$ channel is slightly larger than in the $2 \mathrm{HDM}$ type I. It is clear from the lower panels of Fig. 2 that before the $W^{ \pm} A^{0}$ threshold, $H^{ \pm} \rightarrow \tau^{ \pm} \nu_{\tau}$ is the dominant decay mode and it is amplified by taking large $\tan \beta$. In fact, such a large $\tan \beta$ not only enhances the $\tau \nu_{\tau}$ channel but also reduces $H^{ \pm} \rightarrow c b, c s, t b$ modes, which are all proportional to $\cot \beta$. After crossing $W^{ \pm} A^{0}$ threshold, $H^{ \pm} \rightarrow W^{ \pm} A^{0}$ becomes the dominant decay mode and taking large $\tan \beta$ can further suppress $H^{ \pm} \rightarrow t b$ and makes $H^{ \pm} \rightarrow W^{ \pm} A^{0}$ even larger. Note that in the alignment limit $\cos (\beta-\alpha)=0$, the coupling $H^{ \pm} W^{\mp} h^{0}$ vanishes while $H^{ \pm} W^{\mp} H^{0}$ is maximal and becomes similar to $H^{ \pm} W^{\mp} A^{0}=g / 2$. Therefore, if $H^{ \pm} \rightarrow W^{\mp} H^{0}$ is kinematically open, it will compete on equal footing with $H^{ \pm} \rightarrow W^{ \pm} A^{0}$.

If $\tan \beta$ increases beyond 20 (45), the $\tau \nu$ mode could become comparable to the $W A$ mode for $m_{H^{ \pm}} \gtrsim 200 \mathrm{GeV}$ and $m_{A^{0}}=100(65) \mathrm{GeV}$ in type $\mathrm{X}$. In such a case, the model would be subject to the current charged Higgs searches via the $\tau \nu$ mode. In the following, we will concentrate on a scenario in which the WA is the dominant mode.

The branching fractions for $A^{0}$ are depicted in Fig. 3 as a function of $\tan \beta$ for $m_{A^{0}}=65 \mathrm{GeV}$ in 2HDM type I (left panel) and type X (right panel). In 2HDM type I, all couplings $A^{0} \mathrm{ff}$ are proportional to $\cot \beta$. Therefore, the $\tan \beta$ factorizes out in the branching ratio calculation leading to constant $B\left(A^{0} \rightarrow b \bar{b}, \tau^{+} \tau^{-}, \mu^{+} \mu^{-}\right)$as a function of $\tan \beta$. In the case of type $\mathrm{X}$, the branching ratios $B\left(A^{0} \rightarrow \tau^{+} \tau^{-}, \mu^{+} \mu^{-}\right)$are enhanced for large $\tan \beta$ while $B\left(A^{0} \rightarrow b \bar{b}\right)$ is suppressed. Note for $m_{A^{0}}=$ $100 \mathrm{GeV}$, none of $A^{0} \rightarrow Z^{*} h^{0}$ and $A^{0} \rightarrow W^{\mp *} H^{ \pm}$are open, we observe similar behavior for $B\left(A^{0} \rightarrow f \bar{f}\right)$ in both types I and $\mathrm{X}$.

\section{2. $L H C$ constraint from $t \rightarrow b H^{+} \rightarrow b \tau \nu_{\tau}$}

For direct searches, the LEP Collaborations [6] had searched for charged Higgs pair production via the DrellYan process $e^{+} e^{-} \rightarrow Z / \gamma \rightarrow H^{+} H^{-}$, excluding $M_{H^{ \pm}}<$ $80 \mathrm{GeV}$ (type II) and $M_{H^{ \pm}}<72.5 \mathrm{GeV}$ (type I) at 95\% confidence level. The LHC Collaborations also reported their charged Higgs search results for various mass regions. In the low mass region, the main decay mode is via $t \rightarrow b H^{+}$followed by $H^{ \pm} \rightarrow \tau^{ \pm} \nu_{\tau}$ from CMS [11,12] and ATLAS $[8,9]$. In the high mass region, the main decay mode is $H^{+} \rightarrow t \bar{b}$ from CMS [11,17] and ATLAS [39].

When the charged Higgs mass is below $m_{t}-m_{b}$, it can be abundantly produced in top-quark decays, $t \rightarrow b H^{+}$, followed by charged Higgs decay $H^{+} \rightarrow \tau^{+} \nu_{\tau}$ or $H^{+} \rightarrow W^{+} A^{0}$. The CMS search for $t \rightarrow b H^{+} \rightarrow b\left(\tau^{+} \nu_{\tau}\right)$ [11-13] set limits on $B\left(t \rightarrow b H^{+}\right) \times B\left(H^{+} \rightarrow \tau^{+} \nu_{\tau}\right)$. We rescale their limits to the type-I and type-X $2 \mathrm{HDMs}$ and show the exclusions in $\left(m_{H^{ \pm}}, \tan \beta\right)$ plane. We note that in types I and X the decay width of $t \rightarrow b H^{+}$scales as $\cot ^{2} \beta$,

$$
\begin{aligned}
\Gamma(t & \left.\rightarrow b H^{+}\right) \\
= & \frac{G_{F}}{8 \sqrt{2} \pi} \frac{\left|V_{t b}\right|^{2}}{m_{t}} \lambda^{1 / 2}\left(1, \frac{m_{b}^{2}}{m_{t}^{2}}, \frac{m_{H^{ \pm}}^{2}}{m_{t}^{2}}\right) \\
& \times\left[\left(m_{t}^{2}+m_{b}^{2}\right) \cot ^{2} \beta\left(m_{t}^{2}+m_{b}^{2}-m_{H^{ \pm}}^{2}\right)-4 m_{t}^{2} m_{b}^{2} \cot ^{2} \beta\right],
\end{aligned}
$$

where $\lambda^{1 / 2}\left(1, x^{2}, y^{2}\right) \equiv \sqrt{\left[1-(x+y)^{2}\right]\left[1-(x-y)^{2}\right]}$.

Interpretation of the CMS exclusion region [11-13] in the framework of 2HDM types I and $\mathrm{X}$ in $\left(\tan \beta, m_{H^{ \pm}}\right)$ plane is illustrated in Fig. 4 for both cases: $H^{ \pm} \rightarrow W^{ \pm} A^{0}$ closed and $H^{ \pm} \rightarrow W^{ \pm} A^{0}$ open. ${ }^{3}$ It is clear that for charged Higgs mass $\leq 120 \mathrm{GeV}$ with the $W^{ \pm} A^{0}$ channel closed, $\tan \beta \leq 12$ is excluded. This exclusion is reduced for $m_{H^{ \pm}} \geq 120 \mathrm{GeV}$ due to the fact that $B\left(H^{+} \rightarrow \tau^{+} \nu_{\tau}\right)$ is

${ }^{3}$ Here the results presented in Fig. 4 are consistent with Fig. 3 of Ref. [40], in which the WA mode was not considered. 

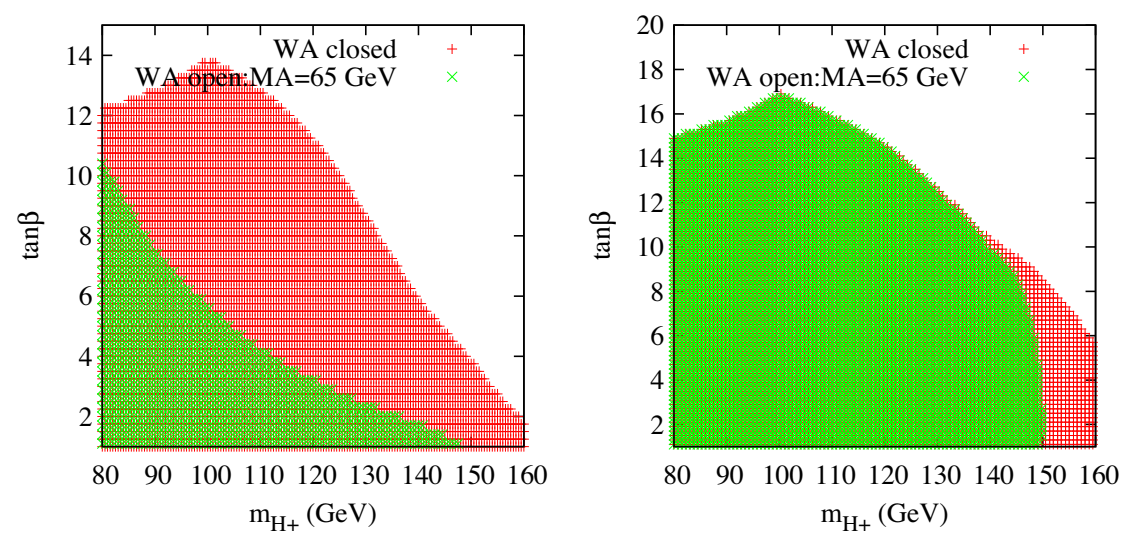

FIG. 4. Interpretation of CMS exclusion regions [11-13] in the 2HDM type I (left panel) and type X (right panel) projected on the plane of $\left(m_{H^{ \pm}}, \tan \beta\right)$. The red points stand for the case the $W A$ mode is closed, while the green points are for the case that the $W A$ mode is open, with $m_{A^{0}}=65 \mathrm{GeV}$.

highly suppressed for $2 \mathrm{HDM}$ type I as $\tan \beta$ increases. On the other hand, when the WA mode is open, the exclusion region in $\left(\tan \beta, m_{H^{ \pm}}\right)$plane is significantly reduced in 2HDM type I. In the case of 2HDM type X, one can see from the right panel that $\tan \beta \leq 6$ is excluded for any value of charged Higgs mass provided that $H^{ \pm} \rightarrow W^{ \pm} A^{0}$ is closed. This limit on $\tan \beta$ is slightly more severe than what we can get from flavor physics (see the above discussion). When $H^{ \pm} \rightarrow W^{ \pm} A^{0}$ is open, starting from $m_{H^{ \pm}} \geq 145 \mathrm{GeV}$ for $m_{A^{0}}=65 \mathrm{GeV}, H^{ \pm} \rightarrow \tau \nu_{\tau}$ mode is suppressed leading to no exclusion for any $\tan \beta$. Below the $W^{ \pm} A^{0}$ threshold, $H^{ \pm} \rightarrow \tau \nu_{\tau}$ channel is still the dominant one, one can see that the green exclusion completely overlaps with the red one in $2 \mathrm{HDM} \mathrm{X}$.

\section{LHC constraint from$$
\boldsymbol{t} \rightarrow \boldsymbol{b H ^ { + }} \rightarrow \boldsymbol{b} W^{+} A^{\mathbf{0}} \rightarrow b W^{+} \mu^{+} \mu^{-}
$$

Recently, the CMS Collaboration [25] also reported the direct search for light charged Higgs via $t \rightarrow b H^{+} \rightarrow$ $b\left(W^{+} A^{0}\right) \rightarrow b\left(l^{+} \nu_{l}\right)\left(\mu^{+} \mu^{-}\right)$with $l=e, \mu[25]$ assuming

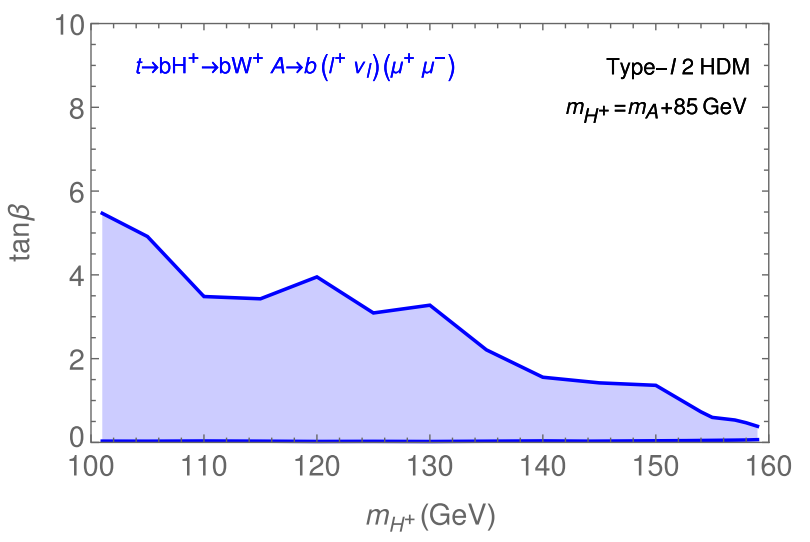

that $H^{ \pm}$decays $100 \%$ into $W^{ \pm} A^{0}$ and $B\left(A^{0} \rightarrow \mu^{+} \mu^{-}\right)=$ $3 \times 10^{-4}$ and set a limit on $B\left(t \rightarrow b H^{+}\right)$. We rescale the CMS limit and interpret it for 2HDM types I and X, which are depicted in Fig. 5. It is clear that the exclusion based on $A^{0} \rightarrow \mu^{+} \mu^{-}$also shows some differences between types I and X. It is easy to see from Fig. 3 that $B\left(A^{0} \rightarrow \mu^{+} \mu^{-}\right)$is only about $2 \times 10^{-4}$ in type I but is as large as $3 \times 10^{-3}$ in type $\mathrm{X}$ for $\tan \beta>3$. Therefore, the excluded region (blue shaded) in Fig. 5 for type $\mathrm{X}$ is much larger than that of type I.

In the rest of this work, we focus on type-I and type-X 2HDMs, in which the charged Higgs mass is much less restricted. In addition, we also focus on the currently allowed parameter space region where $H^{ \pm}$decays dominantly into $W^{ \pm} A^{0}$ via VBF production of same-sign charged Higgs boson pair. This is complementary to the study in Ref. [26].

Before moving to the next section, we make some comments for direct searches of light $H^{0}$ and $A^{0}$ at the LHC. In the (near)-alignment limit, only fermionic

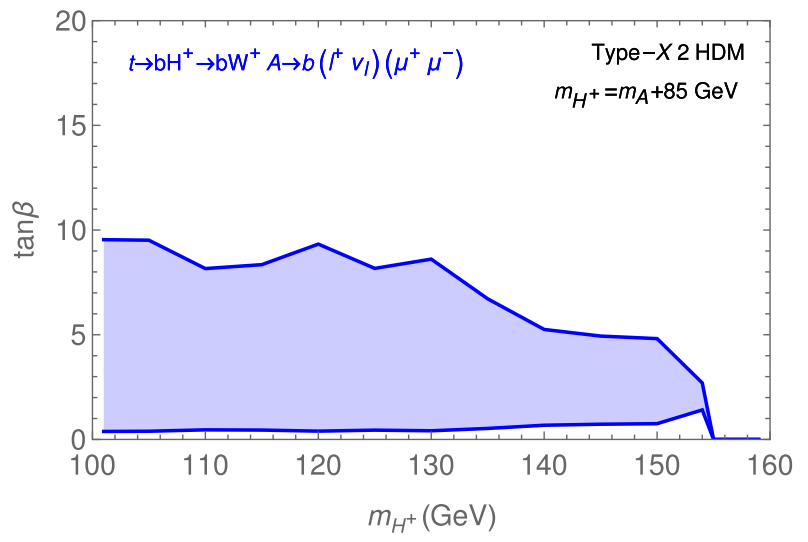

FIG. 5. Exclusions in the parameter space of $\left(m_{H^{ \pm}}, \tan \beta\right)$ for type I (left panel) and for type X (right panel) $2 \mathrm{HDMs}$ obtained by rescaling the observed limits of the CMS results in Ref. [25] based on $t \rightarrow b H^{+} \rightarrow b W^{+} A \rightarrow b\left(l^{+} \nu_{l}\right)\left(\mu^{+} \mu^{-}\right)$. 
production channels $g g \rightarrow H^{0} / A^{0}, p p \rightarrow t \bar{t} H^{0} / A^{0}$, and $p p \rightarrow$ $b \bar{b} H^{0} / A^{0}$ with decay modes $H^{0} / A^{0} \rightarrow b \bar{b}, \tau^{+} \tau^{-}, \mu^{+} \mu^{-}$, and $\gamma \gamma$ are possible to directly detect light $H^{0}$ and $A^{0}[41-44]{ }^{4}$ In type-I and type-X $2 \mathrm{HDMs}$, all of these production channels are proportional to $\cot ^{2} \beta$. Therefore, it is rather challenging to detect both of them for large $\tan \beta$. Besides, it is also hard to distinguish between the $C P$ properties of light $H^{0}$ and $A^{0}$ at the LHC, even if we already observe two different resonance peaks from their fermionic channels. Based on these difficulties, we argue that the process in Eq. (1) can be another way to double check the mass splitting $\Delta m$ between $H^{0}$ and $A^{0}$.

Note that the case of relatively light $C P$ odd $\left(m_{A}<60 \mathrm{GeV}\right)$ is now rather severely constrained by LHC searches. Several dedicated searches can be used to constraint such scenario. The first search is $p p \rightarrow h^{0} \rightarrow$ $A^{0} A^{0} \rightarrow 4 f$ [45-49] which is performed both by ATLAS and CMS and the second one is $p p \rightarrow H^{0} \rightarrow Z A^{0} \rightarrow$ $2 b l^{+} l^{-}$[50]. Even though the $h^{0} A^{0} A^{0}$ coupling can be adjusted to be very small by tuning the parameter $m_{12}^{2}$, this $m_{12}^{2}$ may also violate theoretical and EWPT constraints as well, especially for large mass splitting between $H^{0}$ and $A^{0}$. In this regard, we perform a global scan for the benchmark point $m_{A^{0}} \in[15-60] \mathrm{GeV}$ with $0.97 \leq \sin (\beta-\alpha) \leq 1$ by using the public software 2HDMC, HiggsBounds [51], and HiggsSignal [52]. For such light $C P$ odd, $h^{0}$ can decay with a significant branching ratio into $A^{0} A^{0}$. In addition, the heavy $C P$-even $H^{0}$ can also decay dominantly into $A^{0} Z$ because $H^{0} A^{0} Z$ coupling being proportional to $\sin (\beta-\alpha) \approx 1$. However, we found that the allowed parameter space that survives to the theoretical and EWPT constraints is now almost excluded either by $p p \rightarrow h^{0} \rightarrow A^{0} A^{0} \rightarrow$ $\left\{b \bar{b} b \bar{b}, \mu^{+} \mu^{-} b \bar{b}, \mu^{+} \mu^{-} \tau^{+} \tau^{-}, b \bar{b} \tau^{+} \tau^{-}\right\}$[45-49] or by $p p \rightarrow$ $H^{0} \rightarrow A^{0} Z \rightarrow b \bar{b} l^{+} l^{-}[50]$ searches.

\section{SAME-SIGN CHARGED HIGGS BOSON PAIR PRODUCTION}

\section{A. The behavior of $\boldsymbol{p} \boldsymbol{p} \rightarrow \boldsymbol{H}^{ \pm} \boldsymbol{H}^{ \pm} \boldsymbol{j}_{\boldsymbol{F}} \boldsymbol{j}_{\boldsymbol{F}}$ process}

Recently, the novel process of same-sign charged Higgs boson pair production was proposed in Ref. [26], and especially this process is very sensitive to the mass splitting $\Delta m \equiv m_{H^{0}}-m_{A^{0}}$ in the 2HDMs as it will be shown below. The cross section is enhanced according to the large mass splitting $\Delta m$. This process can be generated via the samesign $W$ boson fusion, $p p \rightarrow W^{ \pm *} W^{ \pm *} j_{F} j_{F} \rightarrow H^{ \pm} H^{ \pm} j_{F} j_{F}$

\footnotetext{
${ }^{4}$ The most stringent constraint from the direct search of light pseudoscalar $A^{0} \rightarrow \tau^{+} \tau^{-}$at the LHC comes from Ref. [42]. If we take $\tan \beta=3$ for type-I and type-X 2HDMs and compare the constraints from Ref. [42] for the process $p p \rightarrow b \bar{b} A^{0}$ with $A^{0} \rightarrow \tau^{+} \tau^{-}$, then the cross sections for type I (type $\mathrm{X}$ ) are about 3 (2) orders smaller than the current constraints for $25<m_{A^{0}}<70 \mathrm{GeV}$. Therefore, we will ignore these constraints in our study.
}

at hadron colliders, where $j_{F}$ denotes the forward and energetic jet directly from the initial parton.

The relation between the mass splitting $\Delta m$ and samesign charged Higgs boson pair production can be understood in the $2 \rightarrow 2$ subprocess $W^{+} W^{+} \rightarrow H^{+} H^{+}$at amplitude level. This subprocess is induced by three t-channel diagrams with $h^{0}, H^{0}$, and $A^{0}$ exchange. In the alignment limit, $\cos (\beta-\alpha)=0$, which is favored by the current Higgs data, the scattering amplitude for

$$
W^{+}\left(p_{1}\right) W^{+}\left(p_{2}\right) \rightarrow H^{+}\left(q_{1}\right) H^{+}\left(q_{2}\right)
$$

is only mediated by $H^{0}$ and $A^{0}$ and is given by

$$
\begin{aligned}
i \mathcal{M}^{H^{0}+A^{0}}= & i g^{2} q_{1} \cdot \epsilon\left(p_{1}\right) q_{2} \cdot \epsilon\left(p_{2}\right)\left[\frac{1}{t-m_{A^{0}}^{2}}-\frac{1}{t-m_{H^{0}}^{2}}\right] \\
& +\left(q_{1} \leftrightarrow q_{2}, t \leftrightarrow u\right) \\
\propto & \Delta m \times \frac{m_{H^{0}}+m_{A^{0}}}{\left(t-m_{H^{0}}^{2}\right)\left(t-m_{A^{0}}^{2}\right)} q_{1} \cdot \epsilon\left(p_{1}\right) q_{2} \cdot \epsilon\left(p_{2}\right) \\
& +\left(q_{1} \leftrightarrow q_{2}, t \leftrightarrow u\right),
\end{aligned}
$$

where $t=\left(p_{1}-q_{1}\right)^{2}$ and $u=\left(p_{1}-q_{2}\right)^{2}$, and $\epsilon\left(p_{1,2}\right)$ are the polarization four-vectors of the incoming $W^{+}$bosons. As it can be seen, the above amplitude is proportional to $\Delta m$.

As indicated by Eq. (9), the production cross section of $p p \rightarrow H^{ \pm} H^{ \pm} j_{F} j_{F}$ scales as the square of the mass splitting $\Delta m$. We quantitatively show this relation by plotting the production cross sections versus $m_{H^{ \pm}}$in Fig. 6 with $\Delta m=100,200$, and $300 \mathrm{GeV}$ at $\sqrt{s}=14 \mathrm{TeV}$ (left panel) and $\sqrt{s}=27 \mathrm{TeV}$ (right panel). It is clear to observe that the cross section is enhanced according to the large mass splitting $\Delta m$. Note that we have used the general twoHiggs-doublet model the Universal FeynRules Output (UFO) model file [53] and employ MadGraph5_aMC@NLO [54] with VBF cut $\eta_{j_{1}} \times \eta_{j_{2}}<0$ and $\left|\Delta \eta_{j j}\right|>2.5$ for the minimum rapidity difference between the forward jet pair to evaluate the cross sections. Furthermore, in order to study the effects of the near-alignment limit on the production cross sections, we list some benchmark points for the relation of cross sections with $\sin (\beta-\alpha)=1,0.95$, 0.9 in Table II at $\sqrt{s}=14 \mathrm{TeV}$ and Table III at $\sqrt{s}=27 \mathrm{TeV}$, respectively.

We stress first that the production cross section $p p \rightarrow$ $H^{ \pm} H^{ \pm} j_{F} j_{F}$ is the same for both $2 \mathrm{HDM}$ types I and X. Only the decay of the charged Higgs bosons will make the process model dependent. The full signal process including decays of $H^{ \pm}, W^{ \pm}, A^{0}$ is given by

$$
\begin{aligned}
p p & \rightarrow W^{ \pm *} W^{ \pm *} j_{F} j_{F} \rightarrow H^{ \pm} H^{ \pm} j_{F} j_{F} \rightarrow\left(W^{ \pm} A^{0}\right)\left(W^{ \pm} A^{0}\right) j_{F} j_{F} \\
& \rightarrow l^{ \pm} \nu_{l}(b \bar{b}) l^{ \pm} \nu_{l}(b \bar{b}) j_{F} j_{F}
\end{aligned}
$$

in type-I 2HDM and 

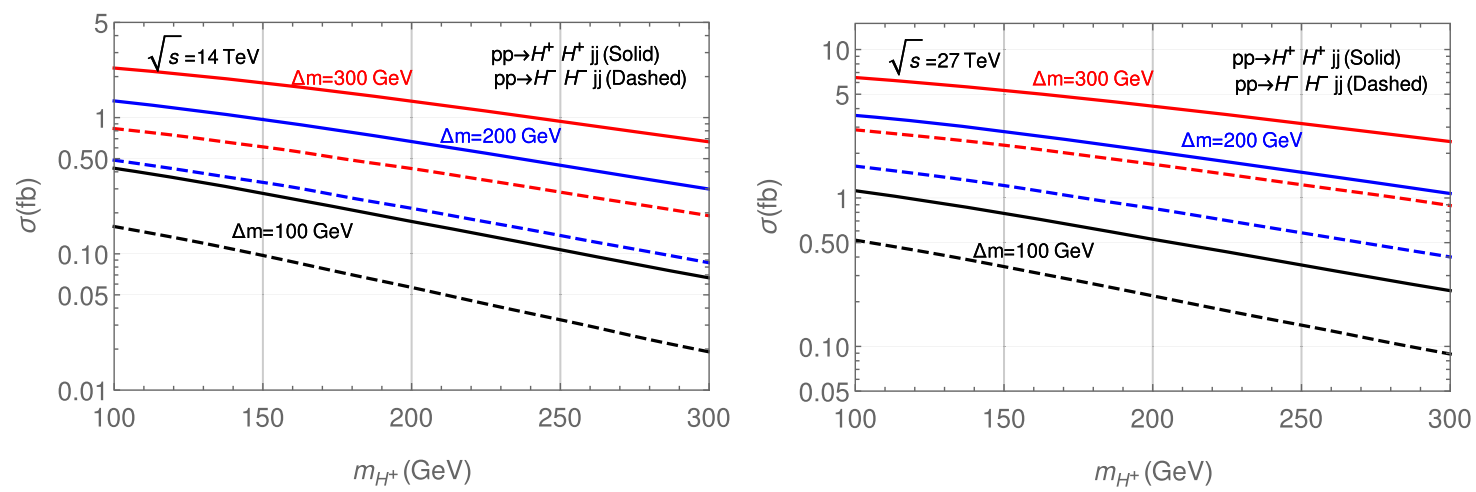

FIG. 6. The production cross sections of $p p \rightarrow H^{+} H^{+} j_{F} j_{F}$ (solid line) and $p p \rightarrow H^{-} H^{-} j_{F} j_{F}$ (dashed line) versus $m_{H^{ \pm}}$at $\sqrt{s}=$ $14 \mathrm{TeV}$ (left panel) and $\sqrt{s}=27 \mathrm{TeV}$ (right panel), for $\Delta m=100 \mathrm{GeV}$ (black), $200 \mathrm{GeV}$ (blue), and $300 \mathrm{GeV}$ (red). Notice the VBF cut $\eta_{j_{1}} \times \eta_{j_{2}}<0$ and $\left|\Delta \eta_{j j}\right|>2.5$ for the minimum rapidity difference between the forward jet pair are applied.

$$
\begin{aligned}
p p & \rightarrow W^{ \pm *} W^{ \pm *} j_{F} j_{F} \rightarrow H^{ \pm} H^{ \pm} j_{F} j_{F} \rightarrow\left(W^{ \pm} A^{0}\right)\left(W^{ \pm} A^{0}\right) j_{F} j_{F} \\
& \rightarrow l^{ \pm} \nu_{l}\left(\tau^{+} \tau^{-}\right) l^{ \pm} \nu_{l}\left(\tau^{+} \tau^{-}\right) j_{F} j_{F}
\end{aligned}
$$

in type-X 2HDM. We advocate that the novel signatures including the combination of a pair of same-sign dileptons $\left(l^{ \pm} l^{ \pm}\right)$, a forward and energetic jet pair $\left(j_{F} j_{F}\right)$, and two pairs of bottom quarks $(b \bar{b})$ or tau leptons $\left(\tau^{+} \tau^{-}\right)$coming from two light pseudoscalars $A^{0}$ can largely reduce the possible SM backgrounds.

Even in case that the masses of $H^{0}$ and $A^{0}$ are separated wide enough and they can be directly measured from other production channels, the current VBF process is still worthwhile to search for. First, the advantage of this process is that it does not depend on Yukawa couplings, in contrast to direct searches of $H^{0}, A^{0}$, or $H^{ \pm}$. The cross section of this process is a function of mainly $m_{H^{ \pm}}, \Delta M$ in the limit $\sin (\beta-\alpha)=1$. Therefore, if no such process is observed, it can exclude the charged Higgs mass or mass correlation between $m_{H^{0}}$ and $m_{A^{0}}$. Second, since $H^{0}$ is difficult to be discovered in the (near)-alignment limit $(\sin (\beta-\alpha) \approx 1)$ in type-I or type-X 2 HDM, this process can imply the mass of $H^{0}$. Nevertheless, this is only true in 2HDMs. If the light boson $A^{0}$ can be discovered in the near future, the usefulness of this process is to tell the mass difference between $H^{0}$ and $A^{0}$ even we do not find the heavier boson $H^{0}$. On the other hand, if both $H^{0}$ and $A^{0}$ have been discovered, the usefulness of this process is to tell if the cross section matches the prediction in 2HDM.

\section{B. Signal-background analysis for type-I 2HDM}

The signal process in Eq. (10) is unique with a signature including the combination of a pair of same-sign dileptons $\left(l^{ \pm} l^{ \pm}\right)$, a pair of forward and energetic jets $\left(j_{F} j_{F}\right)$, and two pairs of bottom quarks $(b \bar{b})$ coming from two light pseudoscalar $A^{0}$. There are a few SM backgrounds that

TABLE II. Sum of cross sections for $\sigma\left(p p \rightarrow H^{+} H^{+} j_{F} j_{F}\right)$ and $\sigma\left(p p \rightarrow H^{-} H^{-} j_{F} j_{F}\right)$ (fb) at $\sqrt{s}=14 \mathrm{TeV}$ for $\sin (\beta-\alpha)=1,0.95,0.9$ with the benchmark points $\Delta m=100,200,300 \mathrm{GeV}$ and $m_{H^{ \pm}}=100,200,300 \mathrm{GeV}$. Notice the $\mathrm{VBF}$ cut $\eta_{j_{1}} \times \eta_{j_{2}}<0$ and $\left|\Delta \eta_{j j}\right|>2.5$ for the minimum rapidity difference between the forward jet pair have been applied.

\begin{tabular}{lcccc}
\hline \hline & \multicolumn{3}{c}{$\sigma\left(p p \rightarrow H^{ \pm} H^{ \pm} j_{F} j_{F}\right)(\mathrm{fb})$} \\
\hline$\Delta m(\mathrm{GeV})$ & $m_{H^{ \pm}}(\mathrm{GeV})$ & $\sin (\beta-\alpha)=1$ & $\sin (\beta-\alpha)=0.95$ & $\sin (\beta-\alpha)=0.9$ \\
\hline 100 & 100 & $5.84 \times 10^{-1}$ & $5.43 \times 10^{-1}$ & $5.03 \times 10^{-1}$ \\
& 200 & $2.30 \times 10^{-1}$ & $2.11 \times 10^{-1}$ & $1.95 \times 10^{-1}$ \\
& 300 & $8.57 \times 10^{-2}$ & $7.86 \times 10^{-2}$ & $7.21 \times 10^{-2}$ \\
200 & 100 & 1.81 & 1.59 & 1.39 \\
& 200 & $8.82 \times 10^{-1}$ & $7.66 \times 10^{-1}$ & $6.62 \times 10^{-1}$ \\
& 300 & $3.85 \times 10^{-1}$ & $3.33 \times 10^{-1}$ & $2.85 \times 10^{-1}$ \\
300 & 100 & 3.14 & 2.70 & 2.32 \\
& 200 & 1.75 & 1.49 & 1.26 \\
& 300 & $8.54 \times 10^{-1}$ & $7.21 \times 10^{-1}$ & $6.05 \times 10^{-1}$ \\
\hline \hline
\end{tabular}


TABLE III. Sum of cross sections for $\sigma\left(p p \rightarrow H^{+} H^{+} j_{F} j_{F}\right)$ and $\sigma\left(p p \rightarrow H^{-} H^{-} j_{F} j_{F}\right)$ (fb) at $\sqrt{s}=27 \mathrm{TeV}$ for $\sin (\beta-\alpha)=1,0.95,0.9$ with the benchmark points $\Delta m=100,200,300 \mathrm{GeV}$ and $m_{H^{ \pm}}=100,200,300 \mathrm{GeV}$. Notice the $\mathrm{VBF}$ cut $\eta_{j_{1}} \times \eta_{j_{2}}<0$ and $\left|\Delta \eta_{j j}\right|>2.5$ for the minimum rapidity difference between the forward jet pair have been applied.

\begin{tabular}{lcccc}
\hline \hline & & \multicolumn{3}{c}{$\sigma\left(p p \rightarrow H^{ \pm} H^{ \pm} j_{F} j_{F}\right)(\mathrm{fb})$} \\
\hline$\Delta m(\mathrm{GeV})$ & $m_{H^{ \pm}}(\mathrm{GeV})$ & $\sin (\beta-\alpha)=1$ & $\sin (\beta-\alpha)=0.95$ & $\sin (\beta-\alpha)=0.9$ \\
\hline 100 & 100 & 1.64 & 1.52 & 1.41 \\
& 200 & $7.46 \times 10^{-1}$ & $6.87 \times 10^{-1}$ & $6.34 \times 10^{-1}$ \\
& 300 & $3.26 \times 10^{-1}$ & $2.99 \times 10^{-1}$ & $2.75 \times 10^{-1}$ \\
200 & 100 & 5.24 & 4.59 & 4.00 \\
& 200 & 2.91 & 2.53 & 2.18 \\
& 300 & 1.47 & 1.27 & 1.09 \\
300 & 100 & 9.35 & 8.04 & 6.87 \\
& 200 & 5.84 & 4.97 & 4.20 \\
& 300 & 3.29 & 2.77 & 2.33 \\
\hline \hline
\end{tabular}

can mimic this kind of final states. We consider the following four processes as the main SM backgrounds:

$$
p p \rightarrow t \bar{t} t \bar{t} \rightarrow\left(b W^{+}\right)\left(\bar{b} W^{-}\right)\left(b W^{+}\right)\left(\bar{b} W^{-}\right) \rightarrow l^{ \pm} l^{ \pm} 4 b 4 j
$$

$$
p p \rightarrow t \bar{b} \bar{t} b l^{+} l^{-} \rightarrow\left(b W^{+}\right) \bar{b}\left(\bar{b} W^{-}\right) b l^{+} l^{-} \rightarrow l^{ \pm} l^{+} l^{-} 4 b 2 j
$$

$$
\begin{aligned}
p p & \rightarrow t \bar{t} t \bar{b} \rightarrow\left(b W^{+}\right)\left(\bar{b} W^{-}\right)\left(b W^{+}\right) \bar{b} \rightarrow l^{+} l^{+} 4 b 2 j \\
o r p p & \rightarrow t \bar{t} \bar{t} b \rightarrow\left(b W^{+}\right)\left(\bar{b} W^{-}\right)\left(\bar{b} W^{-}\right) b \rightarrow l^{-} l^{-} 4 b 2 j, \\
p p & \rightarrow t t \bar{b} \bar{b} j j \rightarrow\left(b W^{+}\right)\left(b W^{+}\right) \bar{b} \bar{b} j j \rightarrow l^{+} l^{+} 4 b 2 j \\
\text { or } p p & \rightarrow \bar{t} \bar{t} b b j j \rightarrow\left(\bar{b} W^{-}\right)\left(\bar{b} W^{-}\right) b b j j \rightarrow l^{-} l^{-} 4 b 2 j .
\end{aligned}
$$

All signal and SM background events are simulated at leading order (LO) using MadGraph5_aMC@NLO. ${ }^{5}$ In the following, we choose $m_{H^{ \pm}}=205 \mathrm{GeV}$ and $m_{A^{0}}=$ $65 \mathrm{GeV}$ to illustrate the cut flow under a sequence of selection cuts at $\sqrt{s}=14 \mathrm{TeV}$.

(1) We first identify the forward jet pair $\left(j_{F} j_{F}\right)$ in the VBF-type process and apply the VBF cut $\eta_{j_{1}} \times \eta_{j_{2}}<0$ and $\left|\Delta \eta_{j j}\right|>2.5$ for the minimum rapidity difference between the forward jet pair in MadGraph5_aMC@NLO at parton level for all signal and SM background events. The cross sections for both signal and background events after this preselection cut are shown in the first row of Table IV.

\footnotetext{
${ }^{5}$ The NLO QCD corrections for the signal process in Eq. (10) and background processes in Eq. (12) and (13) have been checked with MadGraph5_aMC@NLO. We assume that the kinematic distributions are only mildly affected by these higher order QCD effects.
}

(2) Then we employ PYTHIA8 [55] for parton showering and hadronization. DELPHES3 [56] with default settings is used for fast detector simulation. ${ }^{6}$ Finally, all events are analyzed with MadanAlysis5 [57]. We require to see a pair of same-sign dileptons $\left(l^{ \pm} l^{ \pm}\right)$ and at least $3 b$ in the event as the trigger with the following sequence of event selection cuts:

$$
\begin{aligned}
N\left(b, l^{ \pm}\right) & \geq 3,2, \quad P_{T}^{l^{ \pm}}>20 \mathrm{GeV}, \quad\left|\eta^{l^{ \pm}}\right|<2.5 \\
P_{T}^{b} & >20 \mathrm{GeV}, \quad\left|\eta^{b}\right|<2.5 .
\end{aligned}
$$

The b-jets are selected with the efficiency as a function of $P_{T}$ as $\epsilon_{b}=0.85 \times \tanh \left(0.0025 \times P_{T}\right) \times$ $\left(25.0 /\left(1+0.063 \times P_{T}\right)\right)$ and the misidentification rate from c-jets and light-jets to b-jets are $P(c \rightarrow$ $b)=0.25 \times \tanh \left(0.018 \times P_{T}\right) \times(1 /(1+0.0013 \times$ $\left.\left.P_{T}\right)\right)$ and $P(j \rightarrow b)=0.01+0.000038 \times P_{T}$, separately. The cross sections for both signal and background events are shown in the second row of Table IV.

(3) The forward jet pair is also required to be energetic with the following selection cuts:

$$
\begin{aligned}
N(j) \geq 2, & p_{T}^{j}>30 \mathrm{GeV}, \\
\left|\eta^{j}\right|<5, & m_{j j}>500 \mathrm{GeV} .
\end{aligned}
$$

\footnotetext{
${ }^{6}$ Notice that we apply the DELPHES 3.4 .1 in the MadGraph5 aMC@NLO. Comparing with the HL-LHC DELPHES card in the most current version DELPHES 3.4.2, they added the conditions $|\eta|<2.5$ and $10<P_{T}<1000 \mathrm{GeV}$ for the same $\tau$-tagging efficiency and light-jet to tau-jet misidentification rate. On the other hand, they also included the $\eta$ dependence with similar $P_{T}$ dependence settings compared with our default version. We expect these changes will only make very mild modifications of our conclusions.
} 
TABLE IV. Cut flow table for the type-I 2HDM signal $p p \rightarrow H^{ \pm} H^{ \pm} j_{F} j_{F}$ with $m_{H^{ \pm}}=205 \mathrm{GeV}, m_{A^{0}}=65 \mathrm{GeV}, \Delta m=200 \mathrm{GeV}$, $\tan \beta=5$, and $\sin (\beta-\alpha)=0.97$, and various backgrounds at $\sqrt{s}=14 \mathrm{TeV}$.

\begin{tabular}{lccccc}
\hline \hline Cross section (fb) & Signal & $t \bar{t} t \bar{t}$ & $t \bar{t} b \bar{b} l^{+} l^{-}$ & $3 t 1 b$ & $2 t 2 b 2 j$ \\
\hline Preselection & $2.07 \times 10^{-2}$ & $4.94 \times 10^{-2}$ & $1.08 \times 10^{-2}$ & $7.74 \times 10^{-5}$ & $8.29 \times 10^{-5}$ \\
\hline$N\left(b, l^{ \pm}\right) \geq 3,2, P_{T}^{b, l^{ \pm}}>20 \mathrm{GeV},\left|\eta^{b, l}\right|<2.5$ & $1.76 \times 10^{-3}$ & $6.17 \times 10^{-3}$ & $9.56 \times 10^{-4}$ & $9.57 \times 10^{-6}$ & $9.81 \times 10^{-6}$ \\
$N(j) \geq 2, P_{T}^{j}>30 \mathrm{GeV}, M_{j j}>500 \mathrm{GeV}$ & $1.46 \times 10^{-3}$ & $5.15 \times 10^{-3}$ & $4.18 \times 10^{-4}$ & $2.88 \times 10^{-6}$ & $4.05 \times 10^{-6}$ \\
$m_{H^{ \pm}}$cuts $M_{b b l^{ \pm}}<250 \mathrm{GeV}$ & $1.41 \times 10^{-3}$ & $3.50 \times 10^{-3}$ & $2.71 \times 10^{-4}$ & $1.85 \times 10^{-6}$ & $2.62 \times 10^{-6}$ \\
$m_{A}$ cuts $50<M_{b b}<90 \mathrm{GeV}$ & $1.30 \times 10^{-3}$ & $1.68 \times 10^{-3}$ & $1.61 \times 10^{-4}$ & $7.58 \times 10^{-7}$ & $1.14 \times 10^{-6}$ \\
\hline \hline
\end{tabular}

The cross sections after this step for both signal and background events are shown in the third row of Table IV.

(4) The kinematical distributions of $M_{b b l^{ \pm}}$and $M_{b b}$ with $m_{H^{ \pm}}=205 \mathrm{GeV}$ and $m_{A^{0}}=65 \mathrm{GeV}$ for the signal and backgrounds are shown in Fig. 7. Note that we have applied all the selection cuts except for $m_{H^{ \pm}}$ and $m_{A^{0}}$ cuts in these two kinematical distributions. The signal distribution of $M_{b b l^{ \pm}}$tends to concentrate in the region of $M_{b b l^{ \pm}}<250 \mathrm{GeV}$ and decreases more rapidly toward the higher $M_{b b l^{ \pm}}$. On the other hand, the background is relatively flat after 150$500 \mathrm{GeV}$. It is also clear to observe the peak shape at $65 \mathrm{GeV}$ in $M_{b b}$ distribution for the signal from the resonance of $A^{0}$. These two behaviors can help us to distinguish between the signal and the background.

(5) Finally, in order to further reduce the contributions from SM backgrounds, the following selection cuts are imposed on both signal and background events. For $m_{H^{ \pm}}$cuts, at least two bottom quarks and a lepton have to satisfy

$$
M_{b b l^{ \pm}} \leq M_{H^{ \pm}}+45 \mathrm{GeV}
$$

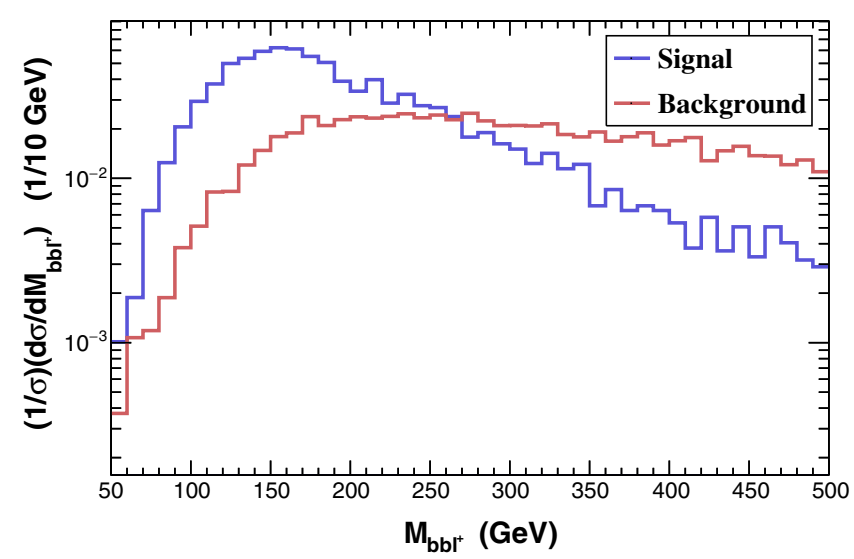

For $m_{A^{0}}$ cuts, at least a pair of bottom quarks is required to be around the mass of $A^{0}$,

$$
m_{A^{0}}-15 \mathrm{GeV} \leq M_{b b} \leq m_{A^{0}}+25 \mathrm{GeV} .
$$

Again, the cross sections for both signal and background events after this sequence of event selection cuts are shown in the last two rows of Table IV.

After all selection cuts, the signal-to-background ratio is almost close to 1 . With a luminosity of $3000 \mathrm{fb}^{-1}$, we expect about four signal and five background events. The major background comes from $t \bar{t} t \bar{t}$ production, while the other backgrounds listed in Table IV are much suppressed.

Even though the signal-to-background ratio is close to one for the analysis at the HL-LHC, the total number of events is small and the fluctuations of SM backgrounds may also be an issue. Since we cannot draw any concrete conclusion for this situation, we further extend the signalbackground analysis to the proposed $27 \mathrm{TeV} p p$ collider (HE-LHC). The SM background cross sections grow faster than the signal one from $\sqrt{s}=14$ to $27 \mathrm{TeV}$. In order to reduce the enhanced background cross sections, both $m_{H^{ \pm}}$ and $m_{A^{0}}$ cuts are tightened relative to those in Eqs. (18) and

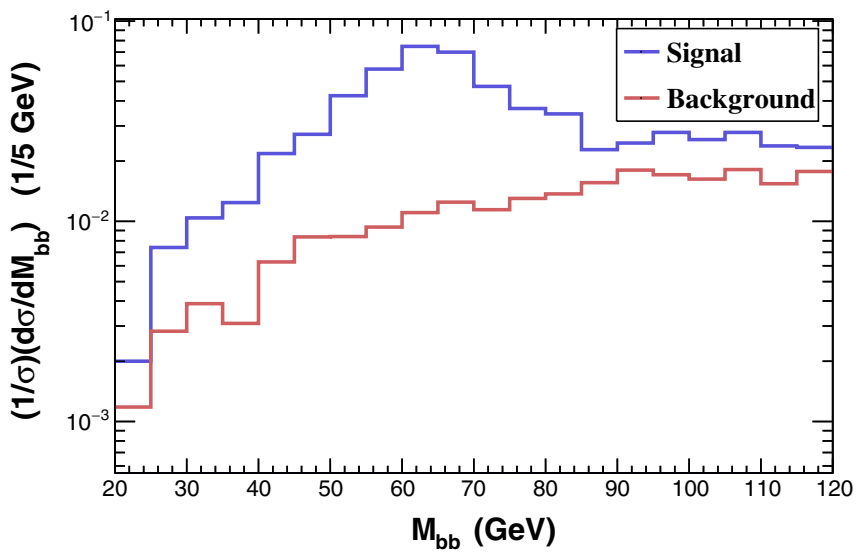

FIG. 7. Invariant mass distributions of $M_{b b l^{ \pm}}$(left panel) and $M_{b b}$ (right panel) for the signal with $m_{H^{ \pm}}=205 \mathrm{GeV}, m_{A^{0}}=65 \mathrm{GeV}$, $\Delta m=200 \mathrm{GeV}, \tan \beta=5$, and $\sin (\beta-\alpha)=0.97$, and the total background at $\sqrt{s}=14 \mathrm{TeV}$. Preselection cuts in Eqs. (16) and (17) are imposed. 
TABLE V. Cut flow table for the type-I 2HDM signal $p p \rightarrow H^{ \pm} H^{ \pm} j_{F} j_{F}$ with $m_{H^{ \pm}}=205 \mathrm{GeV}, m_{A^{0}}=65 \mathrm{GeV}$, $\Delta m=200 \mathrm{GeV}, \tan \beta=5$ and $\sin (\beta-\alpha)=0.97$, and various backgrounds at $\sqrt{s}=27 \mathrm{TeV}$.

\begin{tabular}{lccccc}
\hline \hline Cross section $(\mathrm{fb})$ & Signal & $t \bar{t} t \bar{t}$ & $t \bar{t} b \bar{b} l^{+} l^{-}$ & $3 t 1 b$ & $2 t 2 b 2 j$ \\
\hline Preselection & $6.88 \times 10^{-2}$ & $5.67 \times 10^{-1}$ & $5.60 \times 10^{-2}$ & $2.40 \times 10^{-4}$ & $6.76 \times 10^{-4}$ \\
\hline$N\left(b, l^{ \pm}\right) \geq 3,2, P_{T}^{b, l^{ \pm}}>20 \mathrm{GeV},\left|\eta^{b, l}\right|<2.5$ & $5.15 \times 10^{-3}$ & $5.67 \times 10^{-2}$ & $4.43 \times 10^{-3}$ & $2.44 \times 10^{-5}$ & $6.42 \times 10^{-5}$ \\
$N(j) \geq 2, P_{T}^{j}>30 \mathrm{GeV}, M_{j j}>500 \mathrm{GeV}$ & $4.54 \times 10^{-3}$ & $5.22 \times 10^{-2}$ & $2.49 \times 10^{-3}$ & $9.67 \times 10^{-6}$ & $3.27 \times 10^{-5}$ \\
$m_{H^{ \pm}}$cuts $M_{b b l^{ \pm}}<200 \mathrm{GeV}$ & $4.10 \times 10^{-3}$ & $2.28 \times 10^{-2}$ & $1.08 \times 10^{-3}$ & $4.29 \times 10^{-6}$ & $1.45 \times 10^{-5}$ \\
$m_{A}$ cuts $50<M_{b b}<80 \mathrm{GeV}$ & $3.76 \times 10^{-3}$ & $1.12 \times 10^{-2}$ & $6.09 \times 10^{-4}$ & $1.91 \times 10^{-6}$ & $7.15 \times 10^{-6}$ \\
\hline \hline
\end{tabular}

(19). For $m_{H^{ \pm}}$cuts, at least two bottom quarks and a lepton have to satisfy

$$
M_{b b l^{ \pm}} \leq M_{H^{ \pm}}-5 \mathrm{GeV}
$$

For $m_{A^{0}}$ cuts, at least a pair of bottom quarks is required to be around the mass of $A^{0}$,

$$
\left|M_{b b}-m_{A}\right| \leq 15 \mathrm{GeV} .
$$

Other preselection cuts, given in Eqs. (16) and (17), are the same as before. On the other hand, the shape of kinematical distributions for $M_{b b l^{ \pm}}$and $M_{b b}$ with $m_{H^{ \pm}}=205 \mathrm{GeV}$ and $m_{A^{0}}=65 \mathrm{GeV}$ at $\sqrt{s}=27 \mathrm{TeV}$ for the signal and backgrounds is similar to Fig. 7, so we do not repeat displaying them here. We choose the same-signal benchmark point to illustrate the cut flow under a sequence of selection cuts at $\sqrt{s}=27 \mathrm{TeV}$ in Table V.

Finally, we summarize our signal-background analysis for type-I 2HDM at $\sqrt{s}=27 \mathrm{TeV}$ with luminosity $\mathcal{L}=$ $15 \mathrm{ab}^{-1}$ in Fig. 8. The preselection cuts in Eqs. (16), (17), (20), and (21) are imposed as before. We vary $m_{A^{0}}$ from 63 to $100 \mathrm{GeV}$ with fixed $\sin (\beta-\alpha)=1$ and $\tan \beta=5$ for $\Delta m=m_{H^{0}}-m_{A^{0}}=100 \mathrm{GeV}$ (upper-left panel), $200 \mathrm{GeV}$ (upper-right panel), and $250 \mathrm{GeV}$ (lower panel) in Fig. 8 as the illustrative examples. The black lines are $m_{H^{ \pm}}=m_{H^{0}}$, the blue lines are $m_{H^{ \pm}}=m_{H^{0}}-15 \mathrm{GeV}$, and the red lines are $m_{H^{ \pm}}=m_{H^{0}}+15 \mathrm{GeV}$. We first define the significance by

$$
Z=\sqrt{2 \cdot[(s+b) \cdot \ln (1+s / b)-s]}
$$

where $s$ and $b$ represent the numbers of signal and background events, respectively. According to the production cross sections of same-sign charged Higgs in the right panel of Fig. 6, it is obvious that the cases with moderate mass splittings $\Delta m$ are difficult to be detected even at HE-LHC with high luminosities. The maximum significance is only about $Z=1.2$ for $\Delta m=100 \mathrm{GeV}$. We need other charged Higgs boson production channels to detect this kind of moderate mass splitting $\Delta m$ cases. However, this same-sign charged Higgs boson production channel is sensitive to the cases with large mass splitting $\Delta m$. The average significance is about $Z=3.5$ for $m_{A^{0}}$ from 63 to $100 \mathrm{GeV}$ with $\Delta m=200 \mathrm{GeV}$, and its maximum can reach to more than $Z=4.4$ at $m_{A^{0}}=63 \mathrm{GeV}$. Moreover, the average significance can grow to about $Z=4.5$ for $m_{A^{0}}$ from 63 to $100 \mathrm{GeV}$ with $\Delta m=250 \mathrm{GeV}$, and its maximum can further reach to $Z=5.8$ for $m_{A^{0}} \leq 70 \mathrm{GeV}$.

We further consider a 5\% systematic uncertainty in estimation of the SM background. The significance of the signal is modified to

$$
Z=\sqrt{2 \cdot\left[(s+b) \cdot \ln \left(\frac{(s+b)\left(b+\sigma_{b}^{2}\right)}{b^{2}+(s+b) \sigma_{b}^{2}}\right)-\frac{b^{2}}{\sigma_{b}^{2}} \cdot \ln \left(1+\frac{\sigma_{b}^{2} s}{b\left(b+\sigma_{b}^{2}\right)}\right)\right]},
$$

where $\sigma_{b}$ is the systematic uncertainty of the SM background $b$. We show the effect of including systematic uncertainties as dashed lines in Fig. 8 for comparisons. ${ }^{7}$ The reduction of the systematic uncertainty in future

\footnotetext{
${ }^{7}$ Notice that the $5 \%$ systematic uncertainty that we have assumed in estimation of the SM background is an optimistic choice. Even it is not trivial, this level of systematics might be still achievable at HE-LHC with luminosity $\mathcal{L}=15 \mathrm{ab}^{-1}$.
}

collider experiments is a long shot, but with better understanding of the SM backgrounds and theoretical calculations, a level of less than $10 \%$ systematic uncertainty is not beyond reach. If we take the number of signal and background events with the cross sections shown in the last row of Table $\mathrm{V}, s=56, b=168$ with $15 \mathrm{ab}^{-1}$ integrated luminosity. The significance $Z=4.1$ with $0 \%$ systematic uncertainty, but reduces to $3.4,2.4,1.4$ with $5 \%, 10 \%, 20 \%$ systematic uncertainties. Therefore, one can see that a 

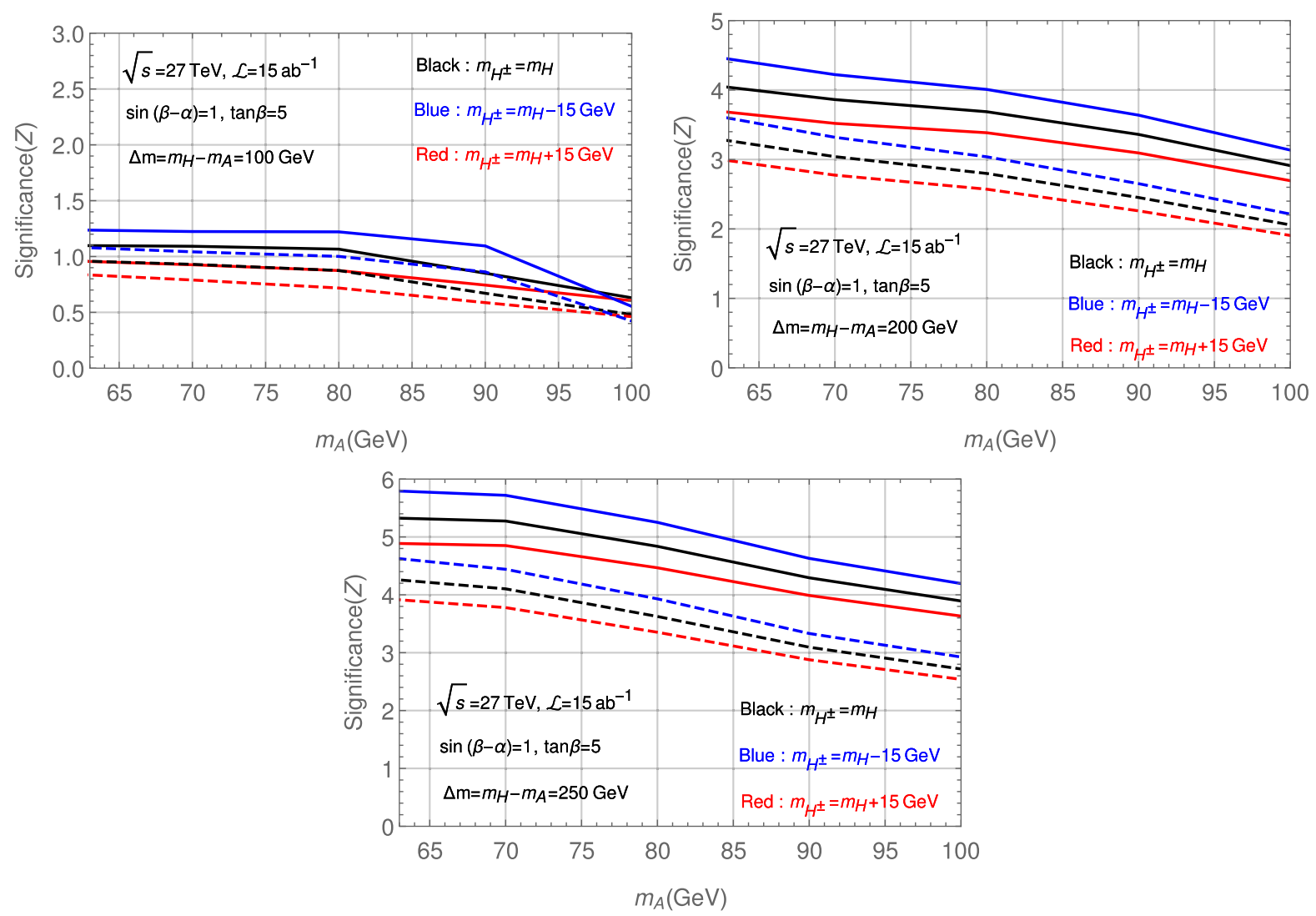

FIG. 8. The significance $Z$ versus $m_{A^{0}}$ from 63 to $100 \mathrm{GeV}$ in type-I $2 \mathrm{HDM}$ at $\sqrt{s}=27 \mathrm{TeV}$ with luminosity $\mathcal{L}=15 \mathrm{ab}^{-1}$. We have fixed $\sin (\beta-\alpha)=1$ and $\tan \beta=5$ with $\Delta m \equiv m_{H^{0}}-m_{A^{0}}=100 \mathrm{GeV}$ (upper-left panel), $200 \mathrm{GeV}$ (upper-right panel), and $250 \mathrm{GeV}$ (lower panel). The dashed lines correspond to additional 5\% systematic uncertainties of the SM background events in Eq. (23).

systematic uncertainty better than $10 \%$ is needed to see a significant excess. In order to preserve a significant excess, it is better to achieve as good as $5 \%$ systematic uncertainty.

\section{Signal-background analysis for type-X 2HDM}

In type-X 2HDM, the major decay of the pseudoscalar $A^{0}$ is $A^{0} \rightarrow \tau \tau$. Therefore, we modify the above signalbackground analysis to two pairs of tau leptons, instead of two pairs of bottom quarks, in the final state. The decay chain is shown in Eq. (11). Therefore, we consider the following set of backgrounds at LO:

$$
\begin{aligned}
& p p \rightarrow t \bar{t} Z j j \rightarrow\left(b W^{+}\right)\left(\bar{b} W^{-}\right)\left(\tau^{+} \tau^{-}\right) j j \rightarrow l^{ \pm} 2 b 3 \tau 2 j, \\
& p p \rightarrow t \bar{t} W^{ \pm} j j \rightarrow\left(b W^{+}\right)\left(\bar{b} W^{-}\right)\left(\tau^{ \pm} \nu_{\tau}\right) j j \rightarrow l^{ \pm} 2 b 2 \tau 2 j,
\end{aligned}
$$

$$
p p \rightarrow W^{ \pm} W^{\mp} Z j j \rightarrow\left(l^{ \pm} \nu_{l}\right)\left(\tau^{\mp} \nu_{\tau}\right)\left(\tau^{+} \tau^{-}\right) j j \rightarrow l^{ \pm} 3 \tau 2 j,
$$

$$
p p \rightarrow W^{ \pm} Z Z j j \rightarrow\left(l^{ \pm} \nu_{l}\right)\left(\tau^{+} \tau^{-}\right)\left(\tau^{+} \tau^{-}\right) j j \rightarrow l^{ \pm} 4 \tau 2 j .
$$

TABLE VI. Cut flow table for the type-X 2HDM signal $p p \rightarrow H^{ \pm} H^{ \pm} j_{F} j_{F}$ with $m_{H^{ \pm}}=205 \mathrm{GeV}, m_{A^{0}}=65 \mathrm{GeV}, \Delta m=200 \mathrm{GeV}$, $\tan \beta=5$, and $\sin (\beta-\alpha)=0.97$, and various backgrounds at $\sqrt{s}=14 \mathrm{TeV}$.

\begin{tabular}{lccccc}
\hline \hline Cross section $(\mathrm{fb})$ & Signal & $t \bar{t} Z j j$ & $t \bar{t} W^{ \pm} j j$ & $W^{ \pm} W^{\mp} Z j j$ & $W^{ \pm} Z Z j j$ \\
\hline Preselection & $2.98 \times 10^{-2}$ & $3.60 \times 10^{-1}$ & $2.44 \times 10^{-1}$ & $3.28 \times 10^{-2}$ & $1.87 \times 10^{-3}$ \\
\hline$N\left(\tau, l^{ \pm}\right) \geq 3,2, P_{T}^{\tau, l^{ \pm}}>20 \mathrm{GeV},\left|\eta^{\tau, l}\right|<2.5$ & $1.23 \times 10^{-3}$ & $7.42 \times 10^{-3}$ & $1.07 \times 10^{-3}$ & $3.89 \times 10^{-4}$ & $9.61 \times 10^{-5}$ \\
$N(j) \geq 2, P_{T}^{j}>30 \mathrm{GeV}, M_{j j}>500 \mathrm{GeV}$ & $9.81 \times 10^{-4}$ & $4.63 \times 10^{-3}$ & $6.19 \times 10^{-4}$ & $1.97 \times 10^{-4}$ & $5.08 \times 10^{-5}$ \\
b-jet veto & $9.15 \times 10^{-4}$ & $1.15 \times 10^{-3}$ & $2.03 \times 10^{-4}$ & $1.71 \times 10^{-4}$ & $4.32 \times 10^{-5}$ \\
$m_{H^{ \pm}}$cut $M_{\tau^{+} \tau^{-}}{ }^{ \pm}<250 \mathrm{GeV}$ & $8.24 \times 10^{-4}$ & $7.52 \times 10^{-4}$ & $9.18 \times 10^{-5}$ & $1.15 \times 10^{-4}$ & $2.98 \times 10^{-5}$ \\
$m_{A^{0}}$ cut $40<M_{\tau^{+}} \tau^{-}<100 \mathrm{GeV}$ & $7.95 \times 10^{-4}$ & $6.28 \times 10^{-4}$ & $5.81 \times 10^{-5}$ & $1.04 \times 10^{-4}$ & $2.73 \times 10^{-5}$ \\
\hline \hline
\end{tabular}



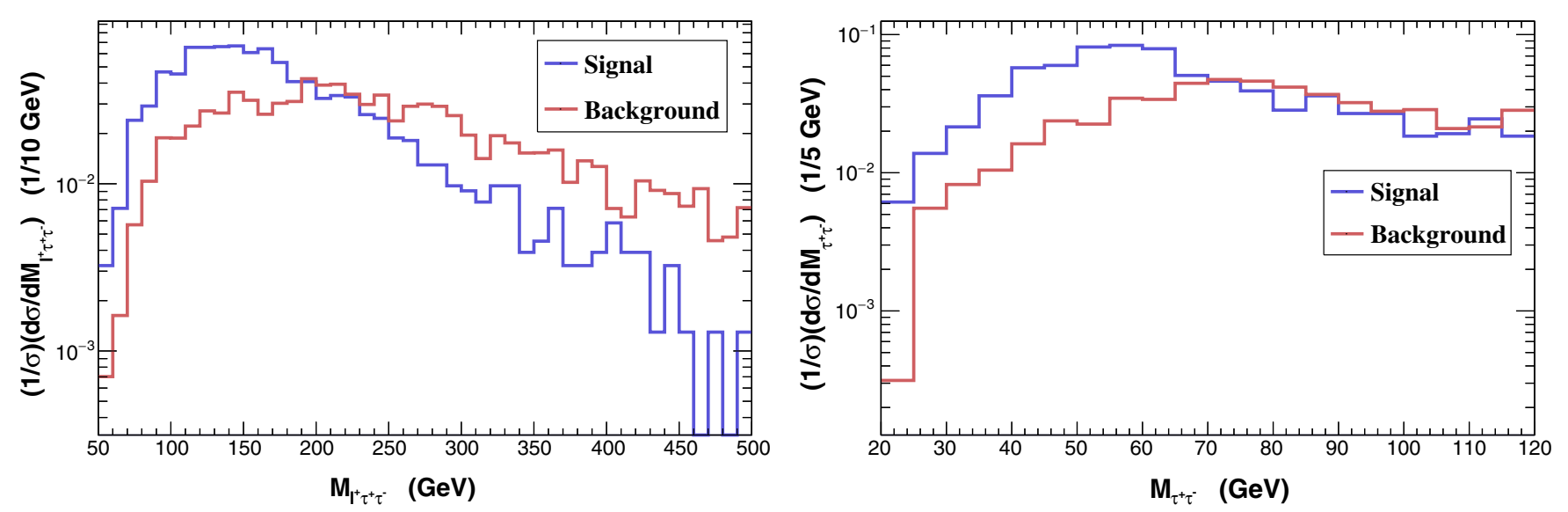

FIG. 9. Invariant mass distributions of $M_{l^{ \pm} \tau^{+} \tau^{-}}$(left panel) and $M_{\tau^{+} \tau^{-}}$(right panel) for the signal with $m_{H^{ \pm}}=205 \mathrm{GeV}$, $m_{A^{0}}=65 \mathrm{GeV}, \Delta m=200 \mathrm{GeV}, \tan \beta=5$, and $\sin (\beta-\alpha)=0.97$, and the total background at $\sqrt{s}=14 \mathrm{TeV}$. Preselection cuts in Eqs. (28)-(30) are imposed.

The extra same-sign charged leptons may come from some cascade decays of the tau leptons, B mesons, or showering. Similarly, the extra tau leptons can also come from B meson cascade decays, showering, or jet misidentification.

Again, we choose $m_{H^{ \pm}}=205 \mathrm{GeV}$ and $m_{A^{0}}=65 \mathrm{GeV}$ to illustrate the cut flow under a sequence of selection cuts.

(1) We apply the same VBF cut $\eta_{j_{1}} \times \eta_{j_{2}}<0$ and $\left|\Delta \eta_{j j}\right|>2.5$ for the minimum rapidity difference between the forward jet pair at parton level for all signal and SM background events. Their cross sections after this preselection cut are shown in the first row of Table VI.

(2) After parton showering and hadronization with PYTHIA8 and detector simulation by DELPHES3, we apply the selections cuts for a pair of same-sign dileptons and at least $3 \tau$,

$$
\begin{aligned}
& N\left(\tau, l^{ \pm}\right) \geq 3,2, \quad P_{T}^{l^{ \pm}}>20 \mathrm{GeV}, \\
& \left|\eta^{l^{ \pm}}\right|<2.5, \quad P_{T}^{\tau}>20 \mathrm{GeV}, \quad\left|\eta^{\tau}\right|<2.5 .
\end{aligned}
$$

Notice we take the hadronic decays of the tau leptons. The tau tagging in DELPHES3 is encoded with the origin of jets from hadronic decay modes of the tau lepton with an efficiency 0.6 and the misidentification rate from light-jet to tau-jet 0.01 . The charge of tau-jet can be determined and reconstructed from the charged pions in the final state according to the algorithm inside DELPHES3. The cross sections for both signal and backgrounds are shown in the second row of Table VI.

(3) The forward jet pair is also required to be energetic with the following selection cuts:

$$
\begin{aligned}
N(j) & >2, \quad p_{T}^{j}>30 \mathrm{GeV}, \quad\left|\eta^{j}\right|<5, \\
m_{j j} & >500 \mathrm{GeV} .
\end{aligned}
$$

The cross sections after this step for both signal and backgrounds are shown in the third row of Table VI.

(4) Since the major background comes from the $t \bar{t}$ associated processes, we apply b-jet veto to suppress background events,

$$
N(b)=0 \quad \text { with } \quad P_{T}^{b}>20 \mathrm{GeV}, \quad\left|\eta^{b}\right|<2.5 .
$$

TABLE VII. Cut flow table for the type-X 2HDM signal $p p \rightarrow H^{ \pm} H^{ \pm} j_{F} j_{F}$ with $m_{H^{ \pm}}=205 \mathrm{GeV}, m_{A^{0}}=65 \mathrm{GeV}, \Delta m=200 \mathrm{GeV}$, $\tan \beta=5$, and $\sin (\beta-\alpha)=0.97$, and various backgrounds at $\sqrt{s}=27 \mathrm{TeV}$.

\begin{tabular}{lccccc}
\hline \hline Cross section $(\mathrm{fb})$ & Signal & $t \bar{t} Z j j$ & $t \bar{t} W^{ \pm} j j$ & $W^{ \pm} W^{\mp} Z j j$ & $W^{ \pm} Z Z j j$ \\
\hline Preselection & $9.93 \times 10^{-2}$ & 2.51 & 1.49 & $1.51 \times 10^{-1}$ & $8.62 \times 10^{-3}$ \\
\hline$N\left(\tau, l^{ \pm}\right) \geq 3,2, P_{T}^{\tau, l^{ \pm}}>20 \mathrm{GeV},\left|\eta^{\tau, l}\right|<2.5$ & $4.27 \times 10^{-3}$ & $4.96 \times 10^{-2}$ & $6.14 \times 10^{-3}$ & $1.71 \times 10^{-3}$ & $4.04 \times 10^{-4}$ \\
$N(j) \geq 2, P_{T}^{j}>30 \mathrm{GeV}, M_{j j}>500 \mathrm{GeV}$ & $3.71 \times 10^{-3}$ & $3.69 \times 10^{-2}$ & $4.50 \times 10^{-3}$ & $1.08 \times 10^{-3}$ & $2.67 \times 10^{-4}$ \\
b-jet veto & $3.40 \times 10^{-3}$ & $9.23 \times 10^{-3}$ & $1.41 \times 10^{-3}$ & $9.23 \times 10^{-4}$ & $2.19 \times 10^{-4}$ \\
$m_{H^{ \pm}}$cut $M_{\tau^{+} \tau^{-} l^{ \pm}}<200 \mathrm{GeV}$ & $2.75 \times 10^{-3}$ & $4.04 \times 10^{-3}$ & $4.17 \times 10^{-4}$ & $3.94 \times 10^{-4}$ & $1.09 \times 10^{-4}$ \\
$m_{A^{0}}$ cut $40<M_{\tau^{+} \tau^{-}}<70 \mathrm{GeV}$ & $2.35 \times 10^{-3}$ & $2.20 \times 10^{-3}$ & $1.96 \times 10^{-4}$ & $2.29 \times 10^{-4}$ & $6.63 \times 10^{-5}$ \\
\hline \hline
\end{tabular}



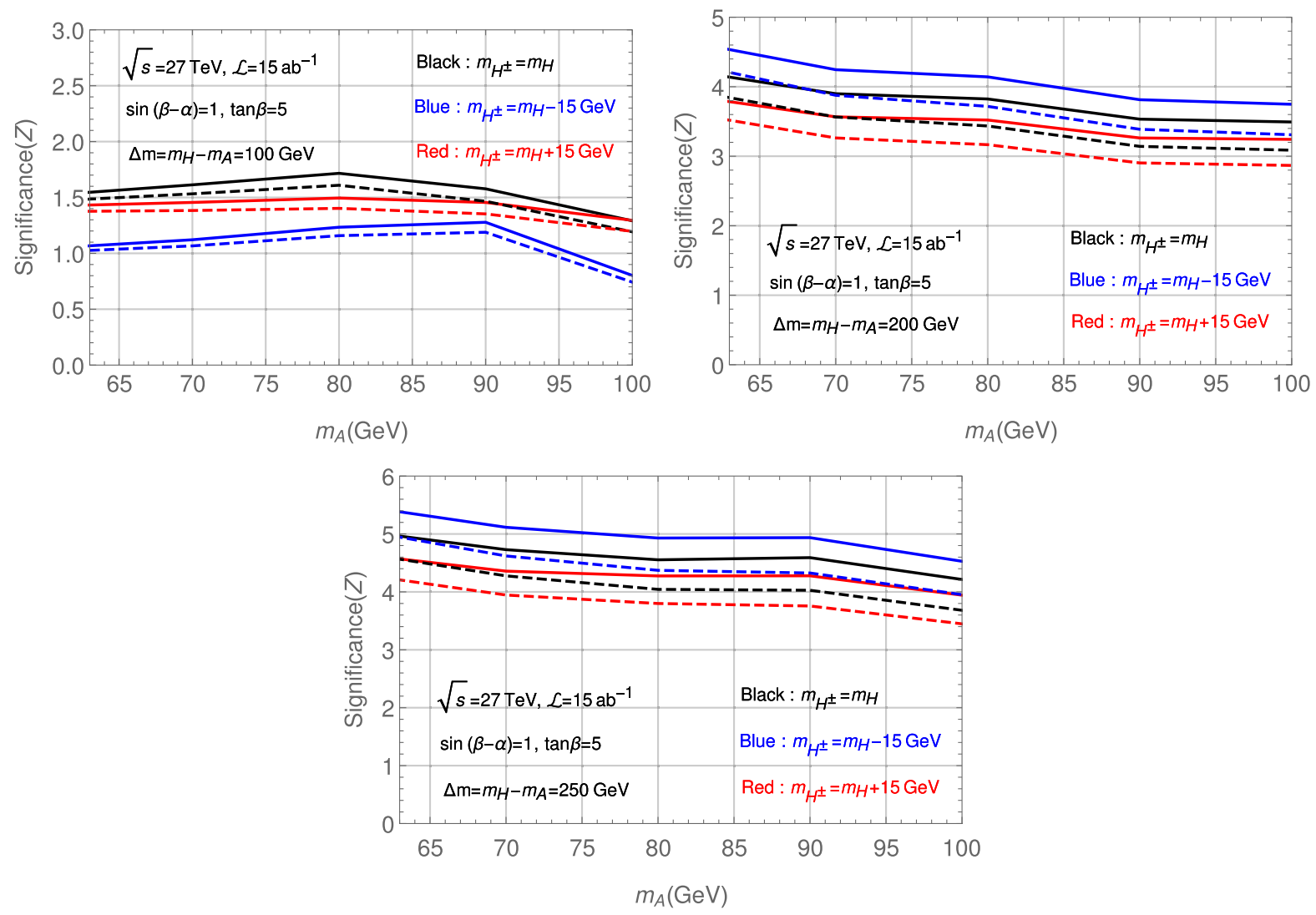

FIG. 10. The same as Fig. 8, but in type-X 2HDM.

The cross sections after this step for both signal and background events are shown in the fourth row of Table VI.

(5) The kinematical distributions of $M_{l^{ \pm} \tau^{+} \tau^{-}}$and $M_{\tau^{+} \tau^{-}}$ with $m_{H^{ \pm}}=205 \mathrm{GeV}$ and $m_{A^{0}}=65 \mathrm{GeV}$ for the signal and backgrounds are shown in Fig. 9. Note that we have applied all the selection cuts except for $m_{H^{ \pm}}$and $m_{A^{0}}$ cuts in these two kinematical distributions. The signal and background distributions of $M_{l^{ \pm} \tau^{+} \tau^{-}}$are similar to $M_{b b l^{ \pm}}$in Fig. 7. However, the peak shape at $65 \mathrm{GeV}$ in $M_{\tau^{+} \tau^{-}}$distribution for the signal from the resonance of $A^{0}$ is not so obvious compared with $M_{b b}$ distribution in Fig. 7. The reason is that the $\tau$-tagging is not as effective as b-tagging. On the other hand, since there are always neutrinos in $\tau$ lepton decays, the $\tau$ lepton cannot be fully reconstructed. This also explains why the shift of fat peak shape from $65 \mathrm{GeV}$ to a slightly lower $M_{\tau^{+} \tau^{-}}$.

(6) Finally, in order to further reduce the contributions from SM backgrounds, the following selection cuts are imposed on both signal and background events. For $m_{H^{ \pm}}$cuts, at least two opposite-sign tau leptons and a lepton have to satisfy

$$
M_{l^{ \pm} \tau^{+} \tau^{-}} \leq M_{H^{ \pm}}+45 \mathrm{GeV}
$$

For the $m_{A^{0}}$ cut, at least a pair of opposite-sign tau leptons is required to around the mass of $A^{0}$,

$$
m_{A^{0}}-25 \mathrm{GeV} \leq M_{\tau^{+} \tau^{-}} \leq m_{A^{0}}+35 \mathrm{GeV} .
$$

The cross sections for both signal and background events after this sequence of event selection cuts are shown in the last two rows of Table VI.

Again, even we can get a good signal-to-background ratio, the total number of events is still small. We further extend the signal-background analysis to the proposed $27 \mathrm{TeV} p p$ collider (HE-LHC). Similar as before, we tighten both $m_{H^{ \pm}}$and $m_{A^{0}}$ cuts relative to those in Eqs. (31) and (32). For $m_{H^{ \pm}}$cuts, at least two tau leptons and a lepton have to satisfy

$$
M_{\tau^{+} \tau^{-} l^{ \pm}} \leq M_{H^{ \pm}}-5 \mathrm{GeV} .
$$

For $m_{A^{0}}$ cuts, at least a pair of opposite-sign tau leptons is required to around the mass of $A^{0},{ }^{8}$

$$
m_{A^{0}}-25 \mathrm{GeV} \leq M_{\tau^{+} \tau^{-}} \leq m_{A^{0}}+5 \mathrm{GeV} .
$$

\footnotetext{
${ }^{8}$ Here we apply an asymmetric mass window cut for $M_{\tau^{+} \tau^{-}}$ based on the shift of peak shape in the right panel of Fig. 9 and in order to veto the pair of opposite-sign tau leptons from the Z-pole.
} 
Other preselection cuts in Eqs. (28)-(30) are imposed, as before. We choose the same-signal benchmark point to illustrate the cut flow under a sequence of selection cuts at $\sqrt{s}=27 \mathrm{TeV}$ in Table VII.

Finally, we summarize the results for signal-background analysis of type-X 2HDM at $\sqrt{s}=27 \mathrm{TeV}$ with luminosity $\mathcal{L}=15 \mathrm{ab}^{-1}$ in Fig. 10. The preselection cuts in Eqs. (28), (29), (33), and (34) are imposed as before. We vary $m_{A^{0}}$ from 63 to $100 \mathrm{GeV}$ with fixed $\sin (\beta-\alpha)=1$ and $\tan \beta=$ 5 for $\Delta m=m_{H^{0}}-m_{A^{0}}=100 \mathrm{GeV}$ (upper-left panel), $200 \mathrm{GeV}$ (upper-right panel), and $250 \mathrm{GeV}$ (lower panel) in Fig. 10 as the illustrative examples. The black lines are $m_{H^{ \pm}}=m_{H^{0}}$, the blue lines are $m_{H^{ \pm}}=m_{H^{0}}-15 \mathrm{GeV}$, and the red lines are $m_{H^{ \pm}}=m_{H^{0}}+15 \mathrm{GeV}$. The maximum significance can reach to about $Z=1.7$ at $m_{A^{0}}=80 \mathrm{GeV}$ for $\Delta m=100 \mathrm{GeV}$. Notice that the mass spectrum with $\Delta m=100 \mathrm{GeV}$ and $m_{H^{ \pm}}=m_{H^{0}}-15 \mathrm{GeV}$ in type-X 2HDM will produce sizable $B\left(H^{ \pm} \rightarrow \tau \nu_{\tau}\right)$ and suppress $B\left(H^{ \pm} \rightarrow W^{ \pm} A^{0}\right)$. That makes reduction of the significance for the blue line in the upper-left panel in Fig. 10. On the other hand, the significance can reach to more than $Z=3$ for $m_{A^{0}}$ from 63 to $100 \mathrm{GeV}$ with $\Delta m=200 \mathrm{GeV}$, and its maximum is about $Z=4.5$ at $m_{A^{0}}=63 \mathrm{GeV}$. Moreover, the significance can grow to more than $Z=4$ for $m_{A^{0}}$ from 63 to $100 \mathrm{GeV}$ with $\Delta m=250 \mathrm{GeV}$, and its maximum can further reach to $Z=5.4$ at $m_{A^{0}}=63 \mathrm{GeV}$. Again, the 5\% systematic errors of the SM background events in Eq. (23) are shown as dashed lines in Fig. 10 for comparisons.

\section{CONCLUSIONS}

Extending the minimal Higgs sector is one of the approaches to address some weakness of the SM. Such extensions can give rise to rich phenomenology. The $2 \mathrm{HDM}$ is one of the most popular extended models in literature. Exploring the whole mass spectrum in 2HDM is undoubtedly an important mission to help us understand the mystery of electroweak symmetry breaking. There are only a few examples that can cover the effects of all new scalar masses in a single process. We have studied a novel process-production of same-sign charged Higgs boson production shown in Eq. (1), which was first proposed in Ref. [26]. It allows one to probe the whole mass spectrum in the 2HDM for some specific mass relations.

We have investigated same-sign charged Higgs boson production via vector-boson fusion at the HL-LHC and HELHC (27 TeV) in type-I and type-X 2HDMs. The dependence of the production cross section on the mass difference $\Delta m \equiv m_{H^{0}}-m_{A^{0}}$ between the heavier scalar boson $H^{0}$ and the pseudoscalar boson $A^{0}$ is studied. The scattering amplitude of the key subprocess $W^{+} W^{+} \rightarrow H^{+} H^{+}$is proportional to $\Delta m$ as shown in Eq. (9), such that the production cross section nearly vanishes in the limit $\Delta m \rightarrow 0$. As we mentioned before, even if the mass splitting $\Delta m$ can be determined by separately measuring
$m_{H^{0}}$ and $m_{A^{0}}$ from other production channels of $H^{0}$ and $A^{0}$, the measurement of same-sign charged Higgs boson production cross section can be used to understand the mass spectrum of the heavier scalar and pseudoscalar bosons in the 2HDMs.

Given the constraints from electroweak precision, B physics, and direct searches at colliders, we have explored the allowed parameter space in $m_{H^{ \pm}}, \tan \beta, \Delta m$. Then we investigated the sensitivity to the allowed parameter space at the HL-LHC and HE-LHC, especially we have made use of the bosonic channel $W^{ \pm} A^{0}$ of the charged Higgs boson, which is complementary to the study in Ref. [26].

In type-I 2HDM, we used the decay channel $H^{ \pm} H^{ \pm} \rightarrow$ $\left(W^{ \pm} A^{0}\right)\left(W^{ \pm} A^{0}\right) \rightarrow\left(l^{ \pm} \nu b \bar{b}\right)\left(l^{ \pm} \nu b \bar{b}\right)$ together with a pair of forward jets to perform the signal-background analysis. At the end, we found about four signal events versus five background events at HL-LHC with luminosity of $3000 \mathrm{fb}^{-1}$ for a typical benchmark point. At the HE-LHC, significance level of 2.6-5.8 can be achieved for $\Delta m=200-250 \mathrm{GeV}$.

On the other hand, in type-X $2 \mathrm{HDM}$, we used the decay channel $H^{ \pm} H^{ \pm} \rightarrow\left(W^{ \pm} A^{0}\right)\left(W^{ \pm} A^{0}\right) \rightarrow\left(l^{ \pm} \nu \tau^{+} \tau^{-}\right) \times$ $\left(l^{ \pm} \nu \tau^{+} \tau^{-}\right)$together with a pair of forward jets to perform the signal-background analysis. At the HL-LHC, we can achieve the signal-to-background ratio equal to 1 , and the number of signal events is about two for a luminosity of $3000 \mathrm{fb}^{-1}$. Nevertheless, at the HE-LHC, the significance can rise to the level of 3.2-5.4 for $\Delta m=200-250 \mathrm{GeV}$.

The main purpose of this study focuses on the search for a possible large mass splitting between the neutral scalar and pseudoscalar through same-sign charged Higgs boson production in $2 \mathrm{HDMs}$ via the vector-boson fusion. It is easy to see that this is not the discovery mode of the charged Higgs because the event rate is much lower than other direct processes, for example, $g b \rightarrow t H^{-}$or $g g \rightarrow t \bar{b} H^{-}$. According to Refs. [12,40] for the search of $g b \rightarrow t H^{-}$or $g g \rightarrow t \bar{b} H^{-}$with $H^{-} \rightarrow \tau^{-} \bar{\nu}_{\tau}$, the constrained region is $\tan \beta \lesssim 2(4)$ in type-I (type-X) $2 \mathrm{HDM}$ for the mass range $160 \leq M_{H^{ \pm}} \leq 180 \mathrm{GeV}$ and there is no constraint for $M_{H^{ \pm}}>180 \mathrm{GeV}$.

Notice that the process in Eq. (1) can be used to distinguish between the charged Higgs boson from a doublet and a triplet. Here, we take the $Y=2$ triplet model (type-II seesaw) as an example. In this model, the triplet VEV is highly constrained from electroweak precision measurement to be less than a few $\mathrm{GeV}$ [58-60]. On the other hand, both $m_{H^{ \pm}}$and $m_{A^{0}}$ in this model are close to degenerate; therefore, $H^{ \pm} \rightarrow W^{ \pm} A^{0}$ is very suppressed. The observation of such decay would exclude type-II seesaw model.

For the VEV of triplet around $1 \mathrm{GeV}$ and $m_{H^{ \pm}}<$ $400 \mathrm{GeV}$, the three dominant $H^{ \pm}$decay modes, $H^{ \pm} \rightarrow$ $\left\{W^{ \pm} h, W^{ \pm} Z, t b\right\}$, are competitive [58]. If one can reconstruct the $Z / h$ invariant mass in the final state, it would be 
viewed as a clear signal beyond 2HDMs in the alignment limit. In the process in Eq. (1), besides the t-channel $Z$ boson exchange and s-channel doubly charged Higgs contributions for the same-sign charged Higgs boson pair production would also show the differences between the triplet model and 2HDMs.

However, in the case of tiny triplet VEV and high triplet mass scale, $H^{ \pm} \rightarrow\left\{W^{ \pm} h, W^{ \pm} Z\right\}$ and also $H^{ \pm \pm} W^{\mp} W^{\mp}$ couplings would be very suppressed. Therefore, the dominant decay mode of $H^{ \pm}$turns out to be $H^{ \pm} \rightarrow l \nu_{l}$ and the doubly charged Higgs contribution in same-sign charged Higgs boson pair production would be small. Besides, because of the mass degeneracy between $A^{0}$ and $H^{ \pm}$in the $Y=2$ triplet model, we would not have $H^{ \pm} \rightarrow W^{ \pm} A^{0}$ decay mode. In the end, for the case of tiny triplet VEV, even the triplet model can mimic the same-sign charged Higgs boson pair production with $H^{ \pm} \rightarrow \tau \nu_{\tau}$ in $2 \mathrm{HDM}$ as shown in Ref. [26]. The $H^{ \pm} \rightarrow W^{ \pm} A^{0}$ decay mode in this work can help us to distinguish $2 \mathrm{HDMs}$ from the triplet model.

One can also advocate $H^{ \pm} \rightarrow W^{ \pm} A^{0}$ decay channel to distinguish the 2HDM from the MSSM which is a 2HDM of type II. However, because of the MSSM sum rules [61], we have $m_{H \pm}^{2}=m_{A^{0}}^{2}+m_{W}^{2}$ which makes the decay channel $H^{ \pm} \rightarrow W^{ \pm} A^{0}$ not open very often and turns out to be rather small. In fact, in the $\operatorname{MSSM}, \operatorname{Br}\left(H^{ \pm} \rightarrow W^{ \pm} A^{0}\right)$ is very suppressed (less than $10^{-2}$ ) while $\operatorname{Br}\left(H^{ \pm} \rightarrow W^{ \pm} h^{0}\right)$ is of the order of a few percent [62]. Therefore, the dominant decay of $H^{ \pm}$are $\tau \nu$ for low charged Higgs mass and $t b$ for $m_{H^{ \pm}}>m_{t}+m_{b}$. In this case also, the MSSM will mimic the same-sign charged Higgs boson pair production with $H^{ \pm} \rightarrow\left\{\tau \nu_{\tau}, t b\right\}$ in 2HDMs as shown in Ref. [26].

In summary, the process in Eq. (1) can be an additional check of the mass relation between same-sign charged Higgs boson production and $\Delta m$, especially, if the $2 \mathrm{HDM}$ mass spectrum has the following relations:

(i) One light (pseudo)scalar, say $A^{0}$.

(ii) A large mass splitting between two neutral scalars, $\Delta m=\left(m_{H^{0}}-m_{A^{0}}\right)$.

(iii) The charged Higgs mass is above the $W^{ \pm} A^{0}$ threshold.

Then this scenario in the 2HDMs can be either pinned down or ruled out in the future.

\section{ACKNOWLEDGMENTS}

The work of K.C. was supported by the National Science Council of Taiwan under Grants No. MOST105-2112-M-007-028-MY3 and No. MOST-107-2112M-007-029-MY3. The work of A. A. was supported in part by the Moroccan Ministry of Higher Education and Scientific Research under Contract No. N'PPR/2015/6. A. A. would like to thank NCTS for hospitality, where this work was done.
[1] T. D. Lee, Phys. Rev. D 8, 1226 (1973).

[2] G. C. Branco, P. M. Ferreira, L. Lavoura, M. N. Rebelo, M. Sher, and J. P. Silva, Phys. Rep. 516, 1 (2012).

[3] S. L. Glashow and S. Weinberg, Phys. Rev. D 15, 1958 (1977).

[4] V. D. Barger, R. J. N. Phillips, and D. P. Roy, Phys. Lett. B 324, 236 (1994).

[5] A. G. Akeroyd et al., Eur. Phys. J. C 77, 276 (2017).

[6] G. Abbiendi et al. (ALEPH, DELPHI, L3, OPAL, and LEP Collaborations), Eur. Phys. J. C 73, 2463 (2013).

[7] V. M. Abazov et al. (D0 Collaboration), Phys. Rev. D 80, 051107 (2009).

[8] G. Aad et al. (ATLAS Collaboration), J. High Energy Phys. 03 (2015) 088.

[9] M. Aaboud et al. (ATLAS Collaboration), J. High Energy Phys. 09 (2018) 139.

[10] M. Aaboud et al. (ATLAS Collaboration), Phys. Lett. B 759, 555 (2016).

[11] V. Khachatryan et al. (CMS Collaboration), J. High Energy Phys. 11 (2015) 018.

[12] A. M. Sirunyan et al. (CMS Collaboration), J. High Energy Phys. 07 (2019) 142.
[13] CMS Collaboration, CERN Report No. CMS-PAS-HIG-16031, 2016.

[14] G. Aad et al. (ATLAS Collaboration), Eur. Phys. J. C 73, 2465 (2013).

[15] ATLAS Collaboration, CERN Report No. ATLAS-CONF2016-089, 2016.

[16] M. Aaboud et al. (ATLAS Collaboration), J. High Energy Phys. 11 (2018) 085.

[17] CMS Collaboration, CERN Report No. CMS-PAS-HIG-18004, 2019.

[18] CMS Collaboration, CERN Report No. CMS-PAS-HIG-18015, 2019.

[19] A. Arhrib, R. Benbrik, and S. Moretti, Eur. Phys. J. C 77, 621 (2017).

[20] F. Kling, A. Pyarelal, and S. Su, J. High Energy Phys. 11 (2015) 051.

[21] B. Coleppa, F. Kling, and S. Su, J. High Energy Phys. 12 (2014) 148.

[22] R. Dermisek, E. Lunghi, and A. Raval, J. High Energy Phys. 04 (2013) 063.

[23] A. G. Akeroyd, A. Arhrib, and Q. S. Yan, Eur. Phys. J. C 55, 653 (2008). 
[24] J. Abdallah et al. (DELPHI Collaboration), Eur. Phys. J. C 34, 399 (2004).

[25] A. M. Sirunyan et al. (CMS Collaboration), Phys. Rev. Lett. 123, 131802 (2019).

[26] M. Aiko, S. Kanemura, and K. Mawatari, Phys. Lett. B 797, 134854 (2019).

[27] M. Aoki, S. Kanemura, K. Tsumura, and K. Yagyu, Phys. Rev. D 80, 015017 (2009).

[28] V. D. Barger, J. L. Hewett, and R. J. N. Phillips, Phys. Rev. D 41, 3421 (1990).

[29] B. W. Lee, C. Quigg, and H. B. Thacker, Phys. Rev. D 16, 1519 (1977).

[30] S. Kanemura, T. Kubota, and E. Takasugi, Phys. Lett. B 313, 155 (1993).

[31] S. Kanemura and K. Yagyu, Phys. Lett. B 751, 289 (2015).

[32] M. Tanabashi et al. (Particle Data Group), Phys. Rev. D 98 , 030001 (2018).

[33] H. E. Haber and A. Pomarol, Phys. Lett. B 302, 435 (1993).

[34] M. Misiak and M. Steinhauser, Eur. Phys. J. C 77, 201 (2017).

[35] M. Misiak et al., Phys. Rev. Lett. 114, 221801 (2015).

[36] T. Enomoto and R. Watanabe, J. High Energy Phys. 05 (2016) 002.

[37] J. Haller, A. Hoecker, R. Kogler, K. Mnig, T. Peiffer, and J. Stelzer, Eur. Phys. J. C 78, 675 (2018).

[38] D. Eriksson, J. Rathsman, and O. Stal, Comput. Phys. Commun. 181, 189 (2010).

[39] G. Aad et al. (ATLAS Collaboration), J. High Energy Phys. 03 (2016) 127.

[40] P. Sanyal, Eur. Phys. J. C 79, 913 (2019).

[41] A. M. Sirunyan et al. (CMS Collaboration), J. High Energy Phys. 11 (2018) 161.

[42] A. M. Sirunyan et al. (CMS Collaboration), J. High Energy Phys. 05 (2019) 210.

[43] A. M. Sirunyan et al. (CMS Collaboration), Phys. Lett. B 793, 320 (2019).

[44] ATLAS Collaboration, CERN Report No. ATLAS-CONF2018-025, 2018.

[45] M. Aaboud et al. (ATLAS Collaboration), Phys. Lett. B 790, 1 (2019).
[46] A. M. Sirunyan et al. (CMS Collaboration), Phys. Lett. B 785, 462 (2018).

[47] M. Aaboud et al. (ATLAS Collaboration), J. High Energy Phys. 10 (2018) 031.

[48] A. M. Sirunyan et al. (CMS Collaboration), Phys. Lett. B 795, 398 (2019).

[49] A. M. Sirunyan et al. (CMS Collaboration), J. High Energy Phys. 11 (2018) 018.

[50] M. Aaboud et al. (ATLAS Collaboration), Phys. Lett. B 783, 392 (2018).

[51] P. Bechtle, O. Brein, S. Heinemeyer, O. Stl, T. Stefaniak, G. Weiglein, and K. E. Williams, Eur. Phys. J. C 74, 2693 (2014).

[52] P. Bechtle, S. Heinemeyer, O. Stl, T. Stefaniak, and G. Weiglein, Eur. Phys. J. C 74, 2711 (2014).

[53] C. Degrande, Comput. Phys. Commun. 197, 239 (2015).

[54] J. Alwall, R. Frederix, S. Frixione, V. Hirschi, F. Maltoni, O. Mattelaer, H.-S. Shao, T. Stelzer, P. Torrielli, and M. Zaro, J. High Energy Phys. 07 (2014) 079.

[55] T. Sjostrand, S. Mrenna, and P. Z. Skands, Comput. Phys. Commun. 178, 852 (2008).

[56] J. de Favereau, C. Delaere, P. Demin, A. Giammanco, V. Lemaître, A. Mertens, and M. Selvaggi (DELPHES 3 Collaboration), J. High Energy Phys. 02 (2014) 057.

[57] E. Conte, B. Fuks, and G. Serret, Comput. Phys. Commun. 184, 222 (2013); E. Conte, B. Dumont, B. Fuks, and C. Wymant, Eur. Phys. J. C 74, 3103 (2014); B. Dumont, B. Fuks, S. Kraml, S. Bein, G. Chalons, E. Conte, S. Kulkarni, D. Sengupta, and C. Wymant, Eur. Phys. J. C 75, 56 (2015).

[58] P. F. Perez, T. Han, G. y. Huang, T. Li, and K. Wang, Phys. Rev. D 78, 015018 (2008).

[59] A. Melfo, M. Nemevsek, F. Nesti, G. Senjanovic, and Y. Zhang, Phys. Rev. D 85, 055018 (2012).

[60] A. Arhrib, R. Benbrik, M. Chabab, G. Moultaka, M. C. Peyranere, L. Rahili, and J. Ramadan, Phys. Rev. D 84, 095005 (2011).

[61] J. F. Gunion, H. E. Haber, and J. Wudka, Phys. Rev. D 43, 904 (1991).

[62] A. Djouadi, Phys. Rep. 459, 1 (2008). 